\title{
Bound-preserving discontinuous Galerkin methods for relativistic hydrodynamics
}

\section{Tong Qin ${ }^{1}$, Chi-Wang Shu ${ }^{2}$ and Yang Yang ${ }^{3}$}

\begin{abstract}
In this paper, we develop a discontinuous Galerkin (DG) method to solve the ideal special relativistic hydrodynamics (RHD) and design a bound-preserving (BP) limiter for this scheme by extending the idea in (X. Zhang and C.-W. Shu, Journal of Computational Physics, 229 (2010), 8918-8934). For RHD, the density and pressure are positive and the velocity is bounded by the speed of light. One difficulty in numerically solving the RHD in its conservative form is that the failure of preserving these physical bounds will result in ill-posedness of the problem and blowup of the code, especially in extreme relativistic cases. The standard way in dealing with this difficulty is to add extra numerical dissipation, while in doing so there is no guarantee in maintaining the high order of accuracy. Our BP limiter has the following features. It can theoretically guarantee to preserve the physical bounds for the numerical solution and maintain its designed high order accuracy. The limiter is local to the cell and hence is very easy to implement. Moreover, it renders $L^{1}$-stability to the numerical scheme. Numerical experiments are performed to demonstrate the good performance of this bound-preserving DG scheme. Even though we only discuss the BP limiter for DG schemes, it can be applied to high order finite volume schemes, such as weighted essentially non-oscillatory (WENO) finite volume schemes as well.
\end{abstract}

Keywords: Bound-preserving; Discontinuous Galerkin method; Relativistic hydrodynamics; $L^{1}$-stability

\footnotetext{
${ }^{1}$ Division of Applied Mathematics, Brown University, Providence, RI 02912. E-mail: tong_qin@brown.edu

${ }^{2}$ Division of Applied Mathematics, Brown University, Providence, RI 02912. E-mail: shu@dam.brown.edu. Research supported by AFOSR grant F49550-12-1-0399 and NSF grant DMS1418750 .

${ }^{3}$ Department of Mathematical Sciences, Michigan Technological University, Houghton, MI 49931. E-mail: yyang7@mtu.edu
} 


\section{Introduction}

Relativistic flows are widely used to model high-energy astrophysical phenomena, such as blast waves of supernova explosions, gravitational collapse and accretion, superluminal jets and gamma-ray bursts. When the speed of the flow is near the speed of the light but there is no strong gravitational field involved, the framework of special relativity is accurate to certain extent to describe the physical phenomena. In this paper, we discuss discontinuous Galerkin (DG) methods to solve the two-dimensional special relativistic hydrodynamics, which can be written into a system of conservation laws as below

$$
\mathbf{w}_{t}+\mathbf{f}(\mathbf{w})_{x}+\mathbf{g}(\mathbf{w})_{y}=0
$$

with

$$
\mathbf{w}=\left(\begin{array}{c}
D \\
m \\
n \\
E
\end{array}\right), \quad \mathbf{f}(\mathbf{w})=\left(\begin{array}{c}
D u \\
m u+p \\
n u \\
m
\end{array}\right), \quad \mathbf{g}(\mathbf{w})=\left(\begin{array}{c}
D v \\
m v \\
n v+p \\
n
\end{array}\right)
$$

as well as its one dimensional version. The method can be easily extended to threedimensions, but this is not discussed in the paper. In (1.2), $p, D, m, n$ and $E$ are the thermal pressure, mass density, momentum in the $x$-direction, momentum in the $y$-direction, and energy, respectively. $(u, v)$ is the velocity field of the fluid. Moreover, units are normalized such that the speed of light is $c=1$. If we denote $\rho$ to be the proper rest-mass density, then the conservative variable $\mathbf{w}$ can be written as

$$
\begin{aligned}
& D=\gamma \rho, \\
& m=D h \gamma u, \\
& n=D h \gamma v, \\
& E=D h \gamma-p,
\end{aligned}
$$

where $\gamma=\left(1-u^{2}-v^{2}\right)^{-1 / 2}$ is the Lorentz factor and $h$ is the specific enthalpy. To close the system, we specify an equation of state $h=h(p, \rho)$. For ideal gas

$$
\rho h=\rho+p \Gamma /(\Gamma-1)
$$


with $\Gamma$ being the specific heat ratio, such that $1<\Gamma \leq 2$ (see for example [39]). Moreover, the sound speed is defined as

$$
c_{s}=\sqrt{\frac{\Gamma p}{\rho h}}=\sqrt{\frac{(\Gamma-1)(h-1)}{h}} .
$$

Physically, the density $D$ and pressure $p$ are positive, and the velocity field $(u, v)$ satisfies $u^{2}+v^{2} \leq 1$. Therefore, we define the admissible set to be

$$
G=\left\{\mathbf{w}: D>0, p(\mathbf{w})>0, u(\mathbf{w})^{2}+v(\mathbf{w})^{2} \leq 1\right\}
$$

By (1.7), it is easy to see that $h>1$, which further yields $0<c_{s} \leq 1$. It is demonstrated in $[24]$ that $G$ is convex and can be represented as

$$
G=\left\{\mathbf{w}: D>0, E>\sqrt{D^{2}+m^{2}+n^{2}}\right\} .
$$

In order to update the flux in the computation, we further need the inverse of (1.3)-(1.6) from the conservative vector $\mathbf{w}$ to the primitive vector $\mathbf{u}=\{\rho, u, v, p\}$. Unlike its Newtonian counterpart, in RHD we do not have an explicit formula for this inverse map. By (1.6), (1.7) and the definition of $\gamma$, we can derive the nonlinear equation satisfied by the pressure $p$

$$
f(p ; \mathbf{w}):=E-\frac{p}{\Gamma-1}-D \sqrt{1-\frac{m^{2}+n^{2}}{(E+p)^{2}}}-\frac{m^{2}+n^{2}}{E+p}=0, \quad p \in[0,+\infty)
$$

By a simple calculation one can show that, if $\mathbf{w} \in G$, then $\frac{\partial f}{\partial p}(p ; \mathbf{w})<0$ and the equation (1.9) has a unique positive solution [47]. In practice, once the bounds (1.8) are satisfied by the numerical solution, (1.9) can be solved efficiently by standard root finding methods. After the pressure is obtained, other quantities can be calculated sequentially and directly via (1.3)-(1.7). Therefore, an efficient and effective conversion from the conservative vector to the primitive vector crucially depends on guaranteeing the bound-preserving property (1.8) for the numerical solution, which is the main objective of this paper.

Numerical simulation of RHD has been intensively studied in the last few decades. The first Eulerian method dates back to the early work by Wilson [44, 45], in which the author used explicit finite differencing techniques and a monotonic transport algorithm to discretize 
the advection terms of the RHD equations. They applied the Von Neumann-Richtmyer artificial viscosity method $[26,33]$ to handle the shock waves. Though this procedure remained standard through the 1980's, it turned out to be unable to resolve the extremely strong shock structures that would appear in the ultra-relativistic regime $(\gamma \geq 2)[1]$. A major breakthrough came in 1994 when Martí et al. [19, 18, 20] reformulated the RHD into the conservation form and for the first time introduced the Godunov-type high resolution shock capturing (HRSC) techniques from the classical gas dynamics simulation to that of the RHD. The HRSC techniques produced high-order approximation in the smooth region and were able to capture shocks and steep transients sharply without spurious oscillations. Since then, various Riemann solvers and modern techniques in gas dynamics were extended to the RHD simulation, for example the relativistic Roe solver by Eulderink et al. [11, 12], the HLLE solver extended by Schneider et al. [34] and the recent relativistic HLLC solver carried out by Mignone et al. [24]. Furthermore, Martí and Müller [21] extended the PPM method [6] to the one dimensional RHD and the multidimensional version was accomplished by Mignone et al. [25]. Donat et al. developed a flux splitting method based on the spectral decomposition of the Jacobian matrix in [9]. Kinetic schemes were developed for RHD in $[16,28]$. Besides these, other high order methods were also well studied, for example, Dolezal and Wong introduced the essentially non-oscillatory (ENO) scheme for the RHD in 1995 [8], which was extended by Del Zanna and Bucciantini in [7]. Subsequently, the WENO algorithm was applied to RHD in [40]. The discontinuous Galerkin methods were also applied to general relativistic hydrodynamics by Radice and Rezzolla [31]. More recently, Zhao and Tang applied WENO limiters to the DG schemes in [62] for the special relativistic case. Moreover, the adaptive mesh refinement (AMR) techniques were proved to be powerful and useful in simulating RHD, for example [54, 43, 14] and many software packages have been developed with AMR support and RHD extension, such as the ENZO [27] and RAMSES [41], etc. Additional methods and details can be found in $[52,53,46,61,17]$, and the references therein, as well as the review paper [22]. 
Although the methods mentioned above have been working successfully in most cases, many authors have reported difficulties in maintaining the physical bounds for the numerical approximation, see, e.g. [12, 34, 9, 8, 54, 62], especially in extreme relativistic cases such as flows with large Lorentz factor, strong shocks, low density and pressure. The violation of the physical bounds may lead to the crash of the code. Actually, a slight violation of the bound $E>\sqrt{D^{2}+m^{2}+n^{2}}$ could give negative pressure, and a more severe violation with $E<\sqrt{m^{2}+n^{2}}$ will even result in the nonexistence of the solution to (1.9), see [34]. Negative pressure ruins the characteristic decomposition in the HRSC methods or kills the Roe solver (see [12]), which all require the square root of the pressure to calculate the speed of the sound. All these could make the code crash in practice. Several ways have been adopted in the literature to get around this problem. For example, in [21], in order to avoid numerical difficulties, the authors set the initial physical quantities, such as the internal energy and pressure, a small number away from zero. In [54], the authors monitor the physical bounds at every time step and once they are broken the calculation will be repeated under a smaller CFL condition with more diffusive schemes. However, these ad hoc techniques are not guaranteed to cure the problem, especially for higher order schemes, and even if they do, the high order accuracy may no longer be maintained.

Physically bound preserving high order numerical methods for conservation laws have been actively studied in the last few years. In 2010, the genuinely maximum-principlesatisfying high order DG and finite volume schemes were constructed in [55] by Zhang and Shu. Subsequently, this technique has been successfully extended to compressible Euler equations without or with source terms [56, 57], shallow water equations [48], and hyperbolic equations with $\delta$-singularities [50]. We also refer the reader to the survey paper [58]. Besides this, a parametrized maximum principle preserving flux limiter is developed by $\mathrm{Xu}$ [49]. The approach in [49] works well numerically, for both finite difference and finite volume schemes, but they can be shown to preserve accuracy only up to third order. In the same spirit, for the compressible Euler system, $\mathrm{Hu}$ et al. [15] developed a flux cut-off limiter for the 
finite difference WENO method to preserve the physical constraints, but to maintain the high-order accuracy of the WENO scheme, rather severe restrictions must be assumed as illustrated in the analysis and numerical tests in [15]. Based on the technique in [15], very recently (which we became aware only after the initial submission of this paper), Wu and Tang [47] developed a bound preserving WENO finite difference scheme for the RHD system (1.1).

In this work, we restrict ourselves to the DG method and provide a systematic and rigorous way to fix the physical bound violation problem, by extending the bound preserving technique for gas dynamics in [56]. The DG method was first introduced by Reed and Hill in 1973 [32] and further developed by Cockburn et al. for nonlinear hyperbolic conservation laws in $[4,3,2,5]$. To overcome the oscillations around strong discontinuities, various limiters have been designed, for example the total variation diminishing or total variation bounded (TVD/TVB) limiters in [36, 4] and the WENO limiter in [29]. But for the RHD system in the extreme relativistic cases, the TVD/TVB or the WENO limiter, when applied alone, will not guarantee to preserve the physical bound and the code could still crash. We would like to emphasize that the BP limiter developed in this paper is not meant as a substitution to the TVB or the WENO limiter or other non-oscillatory techniques, but is simply a remedy to help maintain the physical bounds without affecting the high order accuracy. The TVB or WENO limiter could still be used and may still be necessary in cases involving strong shocks, since the BP limiter is not designed to remove oscillations around shocks, especially when these oscillations are not happening near the physical bounds. However, since the main objective of this paper is the discussion on the bound-preserving limiter, we will not discuss in length about the DG method itself or the TVB or WENO limiters in controlling spurious oscillations. Finally, let us remark that, even though we only discuss the bound-preserving limiter for DG schemes in this paper, the same limiter can be applied to high order finite volume schemes, such as WENO finite volume schemes as well.

The organization of this paper is as follows. In section 2 , we study the one-dimensional 
problem, including the details of the DG scheme, the foundation of the limiters, the $L^{1}$ stability of the method and high order time discretizations. In sections 3 and 4, we study the problem in two space dimensions and the implementation of the relativistic axisymmetric jets. Numerical experiments are given in section 5. Finally, we will end in section 6 with concluding remarks and remarks for future work. The proof of two technical lemmas are given in the Appendixes.

\section{Numerical algorithm in one space dimension}

In this section, we proceed to construct the bound-preserving DG scheme to solve the onedimensional relativistic hydrodynamics.

\subsection{The DG scheme}

We consider the one-dimensional version of (1.1) on the spatial domain $[0,1]$ and solve

$$
\mathbf{w}_{t}+\mathbf{f}(\mathbf{w})_{x}=0
$$

where the conservative variable $\mathbf{w}=(D, m, E)^{T}$ is defined in $(1.3),(1.4),(1.6)$ with $\gamma=$ $\left(1-u^{2}\right)^{-1 / 2}$. The flux is $\mathbf{f}(\mathbf{w})=(D u, m u+p, m)^{T}$ and the equation of state is given in (1.7). The admissible set is defined to be

$$
G_{1}=\left\{(D, m, E)^{T}: D>0, E>\sqrt{D^{2}+m^{2}}\right\}
$$

It is easy to see that $G_{1}$ is convex. To construct the scheme, we divide the computational domain $\Omega=[0,1]$ into $N$ cells

$$
0=x_{\frac{1}{2}}<x_{\frac{3}{2}}<\cdots<x_{N+\frac{1}{2}}=1
$$

and denote

$$
I_{j}=\left(x_{j-\frac{1}{2}}, x_{j+\frac{1}{2}}\right), \quad j=1, \cdots, N
$$

as the cells. For simplicity, we consider uniform meshes in this paper, and denote by $\Delta x$ the size of each cell. 
Next, we define

$$
V_{\Delta x}=\left\{\mathbf{v}: \text { each of its components }\left.v_{i}\right|_{I_{j}} \in \mathcal{P}^{k}\left(I_{j}\right), j=1, \cdots, N\right\}
$$

as the finite element space, where $\mathcal{P}^{k}\left(I_{j}\right)$ denotes the space of polynomials in $I_{j}$ of degree at most $k$.

To define the DG scheme, we first multiply (take the inner product with) the equation (2.1) by any smooth function $\mathbf{v}$, integrate over the cell $I_{j}$ to obtain, after a simple integration by parts

$$
\left(\mathbf{w}_{t}, \mathbf{v}\right)_{j}-\left(\mathbf{f}(\mathbf{w}), \mathbf{v}_{x}\right)_{j}+\mathbf{f}_{j+\frac{1}{2}} \mathbf{v}_{j+\frac{1}{2}}-\mathbf{f}_{j-\frac{1}{2}} \mathbf{v}_{j-\frac{1}{2}}=0
$$

where $(\mathbf{w}, \mathbf{v})_{j}=\int_{I_{j}} \mathbf{w} \cdot \mathbf{v} d x, \mathbf{f}_{j+\frac{1}{2}}=\mathbf{f}\left(\mathbf{w}\left(x_{j+\frac{1}{2}}\right)\right)$ and $\mathbf{v}_{j+\frac{1}{2}}=\mathbf{v}\left(x_{j+\frac{1}{2}}\right)$.

Next, we replace the smooth function $\mathbf{v}$ by the test function $\mathbf{v} \in V_{\Delta x}$ and the exact solution $\mathbf{w}$ by the approximate one in $V_{\Delta x}$ (still denoted by $\mathbf{w}$ by abusing the notation). Since the numerical solution $\mathbf{w} \in V_{\Delta x}$ is discontinuous at the point $x_{j+\frac{1}{2}}$, we need to further replace the flux $\mathbf{f}\left(\mathbf{w}\left(x_{j+\frac{1}{2}}\right)\right)$ by the numerical flux $\hat{\mathbf{f}}_{j+\frac{1}{2}}$, a single valued vector, which is defined at the cell interfaces and in general depends on the values of $\mathbf{w}$ from both sides of the interfaces

$$
\hat{\mathbf{f}}_{j+\frac{1}{2}}=\hat{\mathbf{f}}\left(\mathbf{w}\left(x_{j+\frac{1}{2}}^{-}\right), \mathbf{w}\left(x_{j+\frac{1}{2}}^{+}\right)\right) \text {. }
$$

In this paper, we apply the local Lax-Friedrichs fluxes

$$
\hat{\mathbf{f}}_{j+\frac{1}{2}}=\frac{1}{2}\left(\mathbf{f}\left(\mathbf{w}_{j+\frac{1}{2}}^{-}\right)+\mathbf{f}\left(\mathbf{w}_{j+\frac{1}{2}}^{+}\right)-\alpha_{f}^{j+\frac{1}{2}}\left(\mathbf{w}_{j+\frac{1}{2}}^{+}-\mathbf{w}_{j+\frac{1}{2}}^{-}\right)\right),
$$

where $\alpha_{f}^{j+\frac{1}{2}}$ is a positive real number to be chosen by the bound-preserving technique. Other numerical fluxes such as the HLLC flux could of course also be considered, but will not be discussed in this paper. Finally, we also need to replace $\mathbf{v}_{j+\frac{1}{2}}$ and $\mathbf{v}_{j-\frac{1}{2}}$ by the one-sided limits in $I_{j}$.

In summary, the DG scheme for (2.1) is the following: find $\mathbf{w} \in V_{\Delta x}$, such that for any $\mathbf{v} \in V_{\Delta x}$

$$
\left(\mathbf{w}_{t}, \mathbf{v}\right)_{j}-\left(\mathbf{f}(\mathbf{w}), \mathbf{v}_{x}\right)_{j}+\hat{\mathbf{f}}_{j+\frac{1}{2}} \mathbf{v}_{j+\frac{1}{2}}^{-}-\hat{\mathbf{f}}_{j-\frac{1}{2}} \mathbf{v}_{j-\frac{1}{2}}^{+}=0
$$

where $\mathbf{v}_{j+\frac{1}{2}}^{-}=\mathbf{v}\left(x_{j+\frac{1}{2}}^{-}\right)$, which denotes the left limit of the vector $\mathbf{v}$ at $x_{j+\frac{1}{2}}$. Likewise for $\mathbf{v}^{+}$. 


\subsection{Bound-preserving technique}

In this subsection, we use the Euler-forward time discretization and briefly discuss the construction of the bound-preserving limiter, based on [58]. For (2.1), direct usage of high order DG methods may result in the appearance of negative density and pressure, and physically irrelevant velocity, leading to ill-posed problems. Moreover, the code may blow up once physically irrelevant quantities appear. Therefore, we would like to apply BP limiters to the scheme. We denote $\mathbf{w}_{j}^{n}$ and $\overline{\mathbf{w}}_{j}^{n}$ to be the numerical solution and its cell average at time level $n$ in cell $I_{j}$. For simplicity, throughout the paper, if we consider generic numerical solution on the whole computational domain $\Omega$, then the subscript $j$ will be omitted. Suppose the exact solution of equation (2.1) is in $G_{1}$, we are interested in constructing numerical solutions which are also in $G_{1}$. The whole procedure is given below.

\subsubsection{First order scheme}

In the first step, we consider a first order scheme

$$
\begin{aligned}
\mathbf{w}_{j}^{n+1} & =\mathbf{w}_{j}^{n}+\lambda\left(\hat{\mathbf{f}}\left(\mathbf{w}_{j-1}^{n}, \mathbf{w}_{j}^{n}\right)-\hat{\mathbf{f}}\left(\mathbf{w}_{j}^{n}, \mathbf{w}_{j+1}^{n}\right)\right) \\
& =\mathbf{H}\left(\mathbf{w}_{j-1}^{n}, \mathbf{w}_{j}^{n}, \mathbf{w}_{j+1}^{n}, \lambda\right),
\end{aligned}
$$

where $\mathbf{w}_{j}^{n}=\overline{\mathbf{w}}_{j}^{n}$ is a constant in each cell $I_{j}$, and $\lambda=\frac{\Delta t}{\Delta x}$ is the ratio of time and space mesh sizes. By (2.3)

$$
\begin{aligned}
\mathbf{w}_{j}^{n+1} & =\mathbf{w}_{j}^{n}+\frac{\lambda}{2}\left[\mathbf{f}\left(\mathbf{w}_{j-1}^{n}\right)+\mathbf{f}\left(\mathbf{w}_{j}^{n}\right)-\alpha_{f}^{j-\frac{1}{2}}\left(\mathbf{w}_{j}^{n}-\mathbf{w}_{j-1}^{n}\right)\right] \\
& -\frac{\lambda}{2}\left[\mathbf{f}\left(\mathbf{w}_{j}^{n}\right)+\mathbf{f}\left(\mathbf{w}_{j+1}^{n}\right)-\alpha_{f}^{j+\frac{1}{2}}\left(\mathbf{w}_{j+1}^{n}-\mathbf{w}_{j}^{n}\right)\right] \\
& =\frac{\lambda}{2}\left[\alpha_{f}^{j-\frac{1}{2}} \mathbf{w}_{j-1}^{n}+\mathbf{f}\left(\mathbf{w}_{j-1}^{n}\right)\right]+\left(1-\frac{\lambda}{2} \alpha_{f}^{j-\frac{1}{2}}-\frac{\lambda}{2} \alpha_{f}^{j+\frac{1}{2}}\right) \mathbf{w}_{j}^{n}+\frac{\lambda}{2}\left[\alpha_{f}^{j+\frac{1}{2}} \mathbf{w}_{j+1}^{n}-\mathbf{f}\left(\mathbf{w}_{j+1}^{n}\right)\right] \\
& =\frac{\alpha_{f}^{j-\frac{1}{2}} \lambda}{2} \mathbf{H}^{+}\left(\mathbf{w}_{j-1}^{n}, \alpha_{f}^{j-\frac{1}{2}}\right)+\left(1-\frac{\lambda}{2} \alpha_{f}^{j-\frac{1}{2}}-\frac{\lambda}{2} \alpha_{f}^{j+\frac{1}{2}}\right) \mathbf{w}_{j}^{n}+\frac{\alpha_{f}^{j+\frac{1}{2}} \lambda}{2} \mathbf{H}^{-}\left(\mathbf{w}_{j+1}^{n}, \alpha_{f}^{j+\frac{1}{2}}\right), \quad(2.6)
\end{aligned}
$$

where

$$
\mathbf{H}^{+}(\mathbf{w}, \alpha)=\mathbf{w}+\frac{1}{\alpha} \mathbf{f}(\mathbf{w}), \quad \mathbf{H}^{-}(\mathbf{w}, \alpha)=\mathbf{w}-\frac{1}{\alpha} \mathbf{f}(\mathbf{w}) .
$$

We have the following lemma whose proof will be given in Appendix A. 
Lemma 2.1. Suppose $\mathbf{w} \in G_{1}$ and the parameter $\alpha$ satisfies

$$
\alpha \geq F(\mathbf{w})=\frac{|u|(h+1-2 h \tau) \gamma^{2}+\sqrt{\tau^{4}(h-1)^{2}+\tau^{2}(h-1)(h+1-2 h \tau)}}{\gamma^{2}(h+1-2 h \tau)+\tau^{2}(h-1)},
$$

with $\tau=(\Gamma-1) / \Gamma$, then $\mathbf{H}^{ \pm}(\mathbf{w}, \alpha) \in G_{1}$.

Remark 2.1. The same result also holds for the one-dimensional flow with nonzero transverse velocity. The proof is almost the same and is therefore omitted.

Remark 2.2. In the standard local Lax-Friedrichs flux, the parameter $\alpha$ is chosen to be the upper bound for the absolute value of the eigenvalues of the Jacobian $\partial \mathbf{f}(\mathbf{w}) / \partial \mathbf{w}$, which in one dimension is given by (see, e.g. [62])

$$
\alpha_{\text {standard }}=\frac{|u|+c}{1+|u| c}=\frac{\sqrt{1-1 / \gamma^{2}}+\sqrt{(\Gamma-1)(1-1 / h)}}{1+\sqrt{1-1 / \gamma^{2}} \sqrt{(\Gamma-1)(1-1 / h)}}
$$

In Figure 2.1, we plot $\alpha$ in (2.7) for the bound-preserving requirement (denoted by $\alpha_{b p}$ ) and

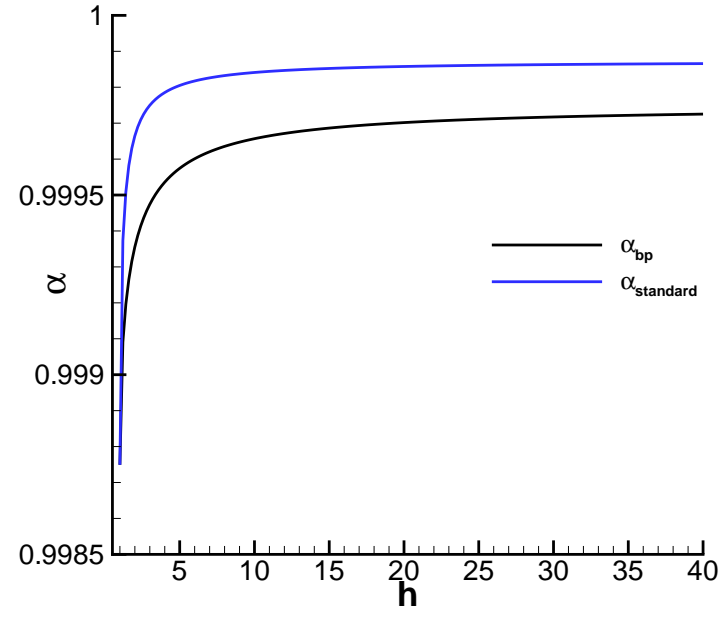

(a) $\gamma=20$

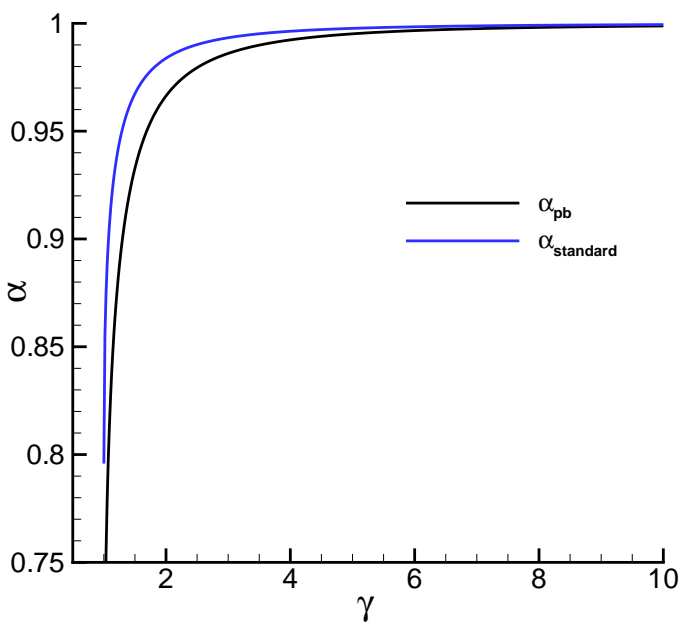

(b) $\mathrm{h}=20$

Figure 2.1: Plots for the lower bound of $\alpha$ in (2.7) for the bound-preserving requirement (denoted by $\alpha_{b p}$ ) and the spectral radius (2.8) of the Jacobian matrix (denoted by $\alpha_{\text {standard }}$ ). The left panel is to keep $\gamma=20$ constant and the right one is to keep $h=20$ constant.

the spectral radius (2.8) of the Jacobian matrix (denoted by $\alpha_{\text {standard }}$ ), for the two situations with $\gamma=20$ as a constant or with $h=20$ as a constant and $\Gamma=5 / 3$ in both plots. We can 
see that the wave speed required for the bound-preserving technique is actually slightly lower (that is, less dissipative!) than the standard wave speed used in the Lax-Friedrichs flux.

In the very recent work of $\mathrm{Wu}$ and Tang [47], which came to our attention after our original submission of this paper, there is a similar result as that in Lemma 2.1. However, the authors of [47] assume $\alpha \geq \alpha_{\text {standard }}$ as defined in (2.8) and show that this condition is sufficient to ensure $\mathbf{H}^{ \pm}(\mathbf{w}, \alpha) \in G_{1}$. Our approach appears to be more constructive and provides a less dissipative solver as shown in Figure 2.1. Moreover, for the first order scheme, the proof in [47] requires a CFL condition $\max _{j} \alpha_{\text {standard }}^{j+\frac{1}{2}} \lambda \leq 1 / 2$. For our approach, from (2.6) we can see that under a more relaxed CFL condition

$$
\max _{j} \alpha_{f}^{j+\frac{1}{2}} \lambda \leq 1
$$

$\mathbf{w}_{j}^{n+1}$ is a convex combination of $\mathbf{H}^{+}\left(\mathbf{w}_{j-1}^{n}, \alpha_{f}^{j-\frac{1}{2}}\right), \mathbf{w}_{j}^{n}$ and $\mathbf{H}^{-}\left(\mathbf{w}_{j+1}^{n}, \alpha_{f}^{j+\frac{1}{2}}\right)$. Therefore, we have the following theorem.

Theorem 2.1. Suppose $\mathbf{w}_{i}^{n} \in G_{1}, i=j-1, j, j+1$, and the parameter $\alpha_{f}^{j+\frac{1}{2}}$ satisfies

$$
\alpha_{f}^{j+\frac{1}{2}} \geq \max \left\{F\left(\mathbf{w}_{j}^{n}\right), F\left(\mathbf{w}_{j+1}^{n}\right)\right\}
$$

with $F$ defined by (2.7), then under the CFL condition (2.9), we have $\mathbf{H}\left(\mathbf{w}_{j-1}^{n}, \mathbf{w}_{j}^{n}, \mathbf{w}_{j+1}^{n}, \lambda\right)=$ $\mathbf{w}_{j}^{n+1} \in G_{1}$.

In the following subsection we will construct high-order bound-preserving DG schemes based on Theorem 2.1.

\subsubsection{High order schemes}

Let $\omega_{i}$ be the Legendre Gauss-Lobatto quadrature weights for the interval $\left[-\frac{1}{2}, \frac{1}{2}\right]$ such that $\sum_{i=0}^{M} \omega_{i}=1$, with $2 M-3 \geq k$, and denote the corresponding Gauss-Lobatto points in cell $I_{j}$ as $\left\{x_{i}^{j}\right\}$. We consider high order schemes and assume $\mathbf{w}^{n}\left(x_{i}^{j}\right) \in G_{1}$ for all $i=0,2, \cdots, M$ and $j=1, \cdots, N$. By taking the test function $\mathbf{v}_{h}=\mathbf{1}$ in (2.4), we have the equation satisfied 
by the numerical cell averages

$$
\overline{\mathbf{w}}_{j}^{n+1}=\overline{\mathbf{w}}_{j}^{n}+\lambda\left(\hat{\mathbf{f}}\left(\mathbf{w}_{j-\frac{1}{2}}^{-}, \mathbf{w}_{j-\frac{1}{2}}^{+}\right)-\hat{\mathbf{f}}\left(\mathbf{w}_{j+\frac{1}{2}}^{-}, \mathbf{w}_{j+\frac{1}{2}}^{+}\right)\right) .
$$

Because of the special choice $2 M-3 \geq k$ of the Gauss-Lobatto quadrature rule, we have

$$
\overline{\mathbf{w}}_{j}^{n}=\sum_{i=0}^{M} \omega_{i} \mathbf{w}_{j}^{n}\left(x_{i}^{j}\right) .
$$

Clearly, $\mathbf{w}_{j}^{n}\left(x_{0}^{j}\right)=\mathbf{w}_{j-\frac{1}{2}}^{+}$and $\mathbf{w}_{j}^{n}\left(x_{M}^{j}\right)=\mathbf{w}_{j+\frac{1}{2}}^{-}$. Therefore, considering $\omega_{0}=\omega_{M}$, we have

$$
\begin{aligned}
\overline{\mathbf{w}}_{j}^{n+1} & =\sum_{i=0}^{M} \omega_{i} \mathbf{w}_{j}^{n}\left(x_{i}^{j}\right)+\lambda\left(\hat{\mathbf{f}}\left(\mathbf{w}_{j-\frac{1}{2}}^{-}, \mathbf{w}_{j-\frac{1}{2}}^{+}\right)-\hat{\mathbf{f}}\left(\mathbf{w}_{j+\frac{1}{2}}^{-}, \mathbf{w}_{j+\frac{1}{2}}^{+}\right)\right) \\
& =\sum_{i=1}^{M-1} \omega_{i} \mathbf{w}_{j}^{n}\left(x_{i}^{j}\right)+\omega_{0}\left[\mathbf{w}_{j-\frac{1}{2}}^{+}+\frac{\lambda}{\omega_{0}}\left(\hat{\mathbf{f}}\left(\mathbf{w}_{j-\frac{1}{2}}^{-}, \mathbf{w}_{j-\frac{1}{2}}^{+}\right)-\hat{\mathbf{f}}\left(\mathbf{w}_{j-\frac{1}{2}}^{+}, \mathbf{w}_{j+\frac{1}{2}}^{-}\right)\right)\right] \\
& +\omega_{0}\left[\mathbf{w}_{j+\frac{1}{2}}^{-}+\frac{\lambda}{\omega_{0}}\left(\hat{\mathbf{f}}\left(\mathbf{w}_{j-\frac{1}{2}}^{+}, \mathbf{w}_{j+\frac{1}{2}}^{-}\right)-\hat{\mathbf{f}}\left(\mathbf{w}_{j+\frac{1}{2}}^{-}, \mathbf{w}_{j+\frac{1}{2}}^{+}\right)\right)\right] \\
& =\sum_{i=1}^{M-1} \omega_{i} \mathbf{w}_{j}^{n}\left(x_{i}^{j}\right)+\omega_{0} \mathbf{H}\left(\mathbf{w}_{j-\frac{1}{2}}^{-}, \mathbf{w}_{j-\frac{1}{2}}^{+}, \mathbf{w}_{j+\frac{1}{2}}^{-}, \frac{\lambda}{\omega_{0}}\right)+\omega_{0} \mathbf{H}\left(\mathbf{w}_{j-\frac{1}{2}}^{+}, \mathbf{w}_{j+\frac{1}{2}}^{-}, \mathbf{w}_{j+\frac{1}{2}}^{+}, \frac{\lambda}{\omega_{0}}\right) .
\end{aligned}
$$

Now Theorem 2.1 yields

$$
\mathbf{H}\left(\mathbf{w}_{j-\frac{1}{2}}^{-}, \mathbf{w}_{j-\frac{1}{2}}^{+}, \mathbf{w}_{j+\frac{1}{2}}^{-}, \frac{\lambda}{\omega_{0}}\right) \in G_{1}, \quad \mathbf{H}\left(\mathbf{w}_{j-\frac{1}{2}}^{+}, \mathbf{w}_{j+\frac{1}{2}}^{-}, \mathbf{w}_{j+\frac{1}{2}}^{+}, \frac{\lambda}{\omega_{0}}\right) \in G_{1},
$$

under the CFL condition $\max _{j} \alpha_{f}^{j+\frac{1}{2}} \lambda<\omega_{0}\left(\right.$ notice that now $\left.\alpha_{f}^{j+\frac{1}{2}} \geq \max \left\{F\left(\mathbf{w}_{j+\frac{1}{2}}^{-}\right), F\left(\mathbf{w}_{j+\frac{1}{2}}^{+}\right)\right\}\right)$. Since $\mathbf{w}_{j}^{n}\left(x_{i}^{j}\right) \in G_{1}$ and $G_{1}$ is a convex set, we have $\overline{\mathbf{w}}_{j}^{n+1} \in G_{1}$.

We would like to emphasize that the cell average $\overline{\mathbf{w}}_{j}^{n+1}$ is shown to be bound-preserving by the original high order DG scheme, before any limiter is applied. Once the cell average is in control, we can modify the numerical solution through a simple scaling limiter

$$
\tilde{\mathbf{w}}_{j}^{n+1}=\overline{\mathbf{w}}_{j}^{n+1}+\theta\left(\mathbf{w}_{j}^{n+1}-\overline{\mathbf{w}}_{j}^{n+1}\right) .
$$

By taking suitable $\theta \in[0,1]$, we will have $\tilde{\mathbf{w}}_{j}^{n+1} \in G_{1}$ at the Gauss-Lobatto points, and $\tilde{\mathbf{w}}_{j}^{n+1}$ is used as the numerical solution at time level $n+1$. For scalar equations, we can prove that this modification does not affect the high order accuracy of the original solution $\mathbf{w}_{j}^{n+1}[58]$. 
Now we give a summary of the complete algorithm. Due to the rounding error, we define

$$
\begin{gathered}
G^{\varepsilon}=\left\{\mathbf{w}=\left(\begin{array}{c}
D \\
m \\
E
\end{array}\right): D \geq \varepsilon, E \geq \sqrt{m^{2}+D^{2}+\varepsilon}\right\}, \\
\partial G^{\varepsilon}=\left\{\mathbf{w}=\left(\begin{array}{c}
D \\
m \\
E
\end{array}\right): D \geq \varepsilon, E=\sqrt{m^{2}+D^{2}+\varepsilon}\right\} .
\end{gathered}
$$

Then the modification of DG solution $\mathbf{w}_{j}^{n}$ is given in the following steps.

- Set up a small number $\varepsilon=10^{-13}$.

- If $\bar{D}_{j}^{n}>\varepsilon$, then proceed to the following steps. Otherwise, $D_{j}^{n}$ is identified as the approximation to vacuum. Therefore, we take $\widetilde{\mathbf{w}}_{j}^{n}=\overline{\mathbf{w}}_{j}^{n}$ as the numerical solution and skip the following steps.

- Modify density in each $I_{j}$ : Compute $b_{j}=\min _{i} D_{j}^{n}\left(x_{i}^{j}\right)$, where $\left\{x_{i}^{j}\right\}$ are the GaussLobatto points in cell $I_{j}$. If $b_{j}<\varepsilon$, then take

$$
\widetilde{D}_{j}^{n}=\bar{D}_{j}^{n}+\theta_{j}^{D}\left(D_{j}^{n}-\bar{D}_{j}^{n}\right)
$$

where

$$
\theta_{j}^{D}=\frac{\bar{D}_{j}^{n}-\varepsilon}{\bar{D}_{j}^{n}-b_{j}} .
$$

Then use $\widetilde{D}_{j}^{n}$ as the new numerical density $D_{j}^{n}$.

- Enforce $E_{j}^{n} \geq \sqrt{\left(m_{j}^{n}\right)^{2}+\left(D_{j}^{n}\right)^{2}+\varepsilon}$ on each Gauss-Lobatto point in each cell $I_{j}$ : Define $\mathbf{q}_{i}^{j}=\mathbf{w}_{j}^{n}\left(x_{i}^{j}\right)$ in cell $I_{j}$. If $\mathbf{q}_{i}^{j} \in G^{\varepsilon}$, then take $\theta_{i}^{j}=1$. Otherwise, take $\theta_{i}^{j}$ to be the root of

$$
\delta_{1}\left((1-t) \overline{\mathbf{w}}_{j}^{n}+t \mathbf{q}_{i}^{j}\right)=0, \quad t \in(0,1)
$$

where $\delta_{1}(\mathbf{w})=E-\sqrt{D^{2}+m^{2}+\varepsilon}$. Then define $\theta_{j}=\min _{i=0, \cdots, M} \theta_{i}^{j}$, and use

$$
\widetilde{\mathbf{w}}_{j}^{n}=\overline{\mathbf{w}}_{j}^{n}+\theta_{j}\left(\mathbf{w}_{j}^{n}-\overline{\mathbf{w}}_{j}^{n}\right),
$$

as the DG approximation in cell $I_{j}$. 
Remark 2.3. In cases where wild data is involved, e.g. the shock heating problem, due to the round-off error, the solution to (2.12) may not strictly make $\left(1-\theta_{i}^{j}\right) \overline{\mathbf{w}}_{j}^{n}+\theta_{i}^{j} \mathbf{q}_{i}^{j} \in G^{\varepsilon}$. In [42], when solving the gas detonation propagation problems with bound preserving DG methods, the authors provide a more robust way to obtain $\theta_{i}^{j}$ without solving any equation. Here, we generalize it to the RHD system. It is straightforward to check that for $t \in(0,1)$,

$$
\delta_{1}\left((1-t) \overline{\mathbf{w}}_{j}^{n}+t \mathbf{q}_{i}^{j}\right) \geq(1-t) \delta_{1}\left(\overline{\mathbf{w}}_{j}^{n}\right)+t \delta_{1}\left(\mathbf{q}_{i}^{j}\right)
$$

Given $D_{j}^{n}\left(x_{i}^{j}\right) \geq \varepsilon, \overline{\mathbf{w}}_{j}^{n} \in G^{\varepsilon}$ and $\delta_{1}\left(\mathbf{q}_{i}^{j}\right)<0$, it is sufficient to require

$$
(1-t) \delta_{1}\left(\overline{\mathbf{w}}_{j}^{n}\right)+t \delta_{1}\left(\mathbf{q}_{i}^{j}\right)=0, \quad \text { i.e., } \quad t=\frac{\delta_{1}\left(\overline{\mathbf{w}}_{j}^{n}\right)}{\delta_{1}\left(\overline{\mathbf{w}}_{j}^{n}\right)-\delta_{1}\left(\mathbf{q}_{i}^{j}\right)}
$$

such that $(1-t) \overline{\mathbf{w}}_{j}^{n}+t \mathbf{q}_{i}^{j} \in G^{\varepsilon}$. Note that the $t$ obtained in this way is in general smaller than that by solving the equation (2.12), but the high order accuracy can still be shown following the same way as in [56].

\subsection{3 $\quad L^{1}$ stability}

Following [51], we can show the $L^{1}$-stability of the numerical scheme, for periodic or compactly supported boundary conditions, with the BP limiter. Since $D^{n}$ is positive, we have, by the conservative property of the DG scheme,

$$
\left\|D^{n}\right\|_{L^{1}}=\int_{\Omega} D^{n}(x) d x=\int_{\Omega} D^{0}(x) d x=\left\|D^{0}\right\|_{L^{1}}
$$

where $\|u\|_{L^{1}}$ is the standard $L^{1}$-norm of $u$ on $\Omega$. Similarly, we can prove $\left\|E^{n}\right\|_{L^{1}}=\left\|E^{0}\right\|_{L^{1}}$. Moreover, it is easy to obtain

$$
m=\frac{u \Gamma}{\Gamma-\sigma} E
$$

where $\sigma=\frac{(\Gamma-1)(h-1)}{h \gamma^{2}} \leq \Gamma-1$. Hence,

$$
\|m\|_{L^{1}} \leq \frac{|u| \Gamma}{\Gamma-\sigma}\|E\|_{L^{1}} \leq \Gamma\|E\|_{L^{1}}
$$

In the last inequality, we use the fact that $|u| \leq 1$. Therefore,

$$
\left\|\mathbf{w}^{n}\right\|_{L^{1}} \leq\left\|D^{n}\right\|_{L^{1}}+(\Gamma+1)\left\|E^{n}\right\|_{L^{1}}=\left\|D^{0}\right\|_{L^{1}}+(\Gamma+1)\left\|E^{0}\right\|_{L^{1}} \leq(\Gamma+1)\left\|\mathbf{w}^{0}\right\|_{L^{1}},
$$

where $\|\mathbf{w}\|_{L^{1}}=\|D\|_{L^{1}}+\|m\|_{L^{1}}+\|E\|_{L^{1}}$. This implies the $L^{1}$-stability of the scheme. 


\subsection{High order time discretizations}

All the previous analyses are based on first-order Euler forward time discretization. We can also use strong stability preserving (SSP) high-order time discretizations to solve the ODE system $\mathbf{w}_{t}=\mathbf{L} \mathbf{w}$. More details of these time discretizations can be found in $[38,37,13]$. In this paper, we use the third order SSP Runge-Kutta method [38]

$$
\begin{aligned}
\mathbf{w}^{(1)} & =\mathbf{w}^{n}+\Delta t \mathbf{L}\left(\mathbf{w}^{n}\right), \\
\mathbf{w}^{(2)} & =\frac{3}{4} \mathbf{w}^{n}+\frac{1}{4}\left(\mathbf{w}^{(1)}+\Delta t \mathbf{L}\left(\mathbf{w}^{(1)}\right)\right), \\
\mathbf{w}^{n+1} & =\frac{1}{3} \mathbf{w}^{n}+\frac{2}{3}\left(\mathbf{w}^{(2)}+\Delta t \mathbf{L}\left(\mathbf{w}^{(2)}\right)\right),
\end{aligned}
$$

and the third order SSP multi-step method [37]

$$
\mathbf{w}^{n+1}=\frac{16}{27}\left(\mathbf{w}^{n}+3 \Delta t \mathbf{L}\left(\mathbf{w}^{n}\right)\right)+\frac{11}{27}\left(\mathbf{w}^{n-3}+\frac{12}{11} \Delta t \mathbf{L}\left(\mathbf{w}^{n-3}\right)\right) .
$$

Since an SSP time discretization is a convex combination of Euler forward, by using the limiter designed in section 2.2, the numerical solution obtained from the full scheme is also in $G_{1}$.

\section{$3 \quad$ Numerical algorithm in two space dimensions}

In this section, we extend the bound-preserving discontinuous Galerkin method to relativistic hydrodynamics in two space dimensions. For simplicity, we use Euler forward time discretization and construct high-order bound-preserving DG schemes to solve (1.1). In this section, we construct the numerical solutions to be in $G$, which is given in (1.8).

For simplicity, we use uniform rectangular meshes. The algorithm including the BP limiter can be easily generalized to unstructured meshes, along the lines in [60]. The cell is defined as $I_{i j}=\left[x_{i-\frac{1}{2}}, x_{i+\frac{1}{2}}\right] \times\left[y_{j-\frac{1}{2}}, y_{j+\frac{1}{2}}\right]$, and the mesh sizes in $x$ and $y$ directions are denoted as $\Delta x$ and $\Delta y$, respectively. At time level $\mathrm{n}$, we approximate the exact solution with a vector of polynomials of degree $k, \mathbf{w}_{i j}^{n}=\left(D_{i j}^{n}, m_{i j}^{n}, n_{i j}^{n}, E_{i j}^{n}\right)^{T}$, and define the cell average $\overline{\mathbf{w}}_{i j}^{n}=\left(\bar{D}_{i j}^{n}, \bar{m}_{i j}^{n}, \bar{n}_{i j}^{n}, \bar{E}_{i j}^{n}\right)^{T}$. Moreover, we denote $\mathbf{w}_{i-\frac{1}{2}, j}^{+}(y), \mathbf{w}_{i+\frac{1}{2}, j}^{-}(y), \mathbf{w}_{i, j-\frac{1}{2}}^{+}(x), \mathbf{w}_{i, j+\frac{1}{2}}^{-}(x)$ as 
the traces of $\mathbf{w}$ on the four edges in cell $I_{i j}$, respectively. More details can be found in [56]. For simplicity, if we consider a generic numerical solution on the whole computational domain at time level $n$, then the subscript $i j$ will be omitted.

In this section, we only consider high-order schemes, and the one satisfied by the cell averages can be written as

$$
\begin{aligned}
\overline{\mathbf{w}}_{i j}^{n+1} & =\overline{\mathbf{w}}_{i j}^{n}+\frac{\Delta t}{\Delta x \Delta y} \int_{y_{j-\frac{1}{2}}}^{y_{j+\frac{1}{2}}} \widehat{\mathbf{f}}\left(\mathbf{w}_{i-\frac{1}{2}, j}^{-}(y), \mathbf{w}_{i-\frac{1}{2}, j}^{+}(y)\right)-\widehat{\mathbf{f}}\left(\mathbf{w}_{i+\frac{1}{2}, j}^{-}(y), \mathbf{w}_{i+\frac{1}{2}, j}^{+}(y)\right) d y \\
& +\frac{\Delta t}{\Delta x \Delta y} \int_{x_{i-\frac{1}{2}}}^{x_{i+\frac{1}{2}}} \widehat{\mathbf{g}}\left(\mathbf{w}_{i, j-\frac{1}{2}}^{-}(x), \mathbf{w}_{i, j-\frac{1}{2}}^{+}(x)\right)-\widehat{\mathbf{g}}\left(\mathbf{w}_{i, j+\frac{1}{2}}^{-}(x), \mathbf{w}_{i, j+\frac{1}{2}}^{+}(x)\right) d x
\end{aligned}
$$

where $\widehat{\mathbf{f}}(\cdot, \cdot)$ and $\widehat{\mathbf{g}}(\cdot, \cdot)$ are one-dimensional numerical fluxes. For this problem, we still use the one-dimensional local Lax-Friedrichs flux. Suppose $(x, y)=\left(x_{i-\frac{1}{2}}, y_{0}\right)$ is a point on the vertical cell interface, at which we have two numerical approximations $\mathbf{w}_{\ell}=\left(D_{\ell}, m_{\ell}, n_{\ell}, E_{\ell}\right)^{T}$ and $\mathbf{w}_{r}=\left(D_{r}, m_{r}, n_{r}, E_{r}\right)^{T}$ from left and right, respectively. Then the local Lax-Friedrichs flux can be written as

$$
\widehat{\mathbf{f}}\left(\mathbf{w}_{\ell}, \mathbf{w}_{r}\right)=\frac{1}{2}\left(\mathbf{f}\left(\mathbf{w}_{\ell}\right)+\mathbf{f}\left(\mathbf{w}_{r}\right)-\alpha_{f}\left(\mathbf{w}_{r}-\mathbf{w}_{\ell}\right)\right)
$$

where $\alpha_{f} \geq \max \left\{F_{1}\left(\mathbf{w}_{\ell}\right), F_{1}\left(\mathbf{w}_{r}\right)\right\}$ with $F_{1}$ is defined by

$$
F_{1}(\mathbf{w})=\frac{|u|(h+1-2 h \tau) \gamma^{2}+\sqrt{\tau^{4}(h-1)^{2}+\tau^{2}(h-1)(h+1-2 h \tau)}}{\gamma^{2}(h+1-2 h \tau)+\tau^{2}(h-1)},
$$

and the constant $\tau=\frac{\Gamma-1}{\Gamma}$. The numerical flux $\widehat{\mathbf{g}}$ can be defined in a similar way with the parameter $\alpha_{g}$ on the horizontal cell interfaces and the corresponding wave speed defined by

$$
F_{2}(\mathbf{w})=\frac{|v|(h+1-2 h \tau) \gamma^{2}+\sqrt{\tau^{4}(h-1)^{2}+\tau^{2}(h-1)(h+1-2 h \tau)}}{\gamma^{2}(h+1-2 h \tau)+\tau^{2}(h-1)},
$$

We extend the definitions of $\mathbf{H}$ in (2.5) to two-dimensional problems and define

$$
\begin{aligned}
& \mathbf{H}_{1}\left(\mathbf{w}_{1}, \mathbf{w}_{2}, \mathbf{w}_{3}, \lambda_{1}\right)=\mathbf{w}_{2}+\lambda_{1}\left(\widehat{\mathbf{f}}\left(\mathbf{w}_{1}, \mathbf{w}_{2}\right)-\widehat{\mathbf{f}}\left(\mathbf{w}_{2}, \mathbf{w}_{3}\right)\right), \\
& \mathbf{H}_{2}\left(\mathbf{w}_{1}, \mathbf{w}_{2}, \mathbf{w}_{3}, \lambda_{2}\right)=\mathbf{w}_{2}+\lambda_{2}\left(\widehat{\mathbf{g}}\left(\mathbf{w}_{1}, \mathbf{w}_{2}\right)-\widehat{\mathbf{g}}\left(\mathbf{w}_{2}, \mathbf{w}_{3}\right)\right),
\end{aligned}
$$

where $\lambda_{1}=\frac{\Delta t}{\Delta x}$ and $\lambda_{2}=\frac{\Delta t}{\Delta y}$. Following the same proof of Theorem 2.1 with some minor changes, we have the following result 
Lemma 3.1. Suppose $\mathbf{w}_{1}, \mathbf{w}_{2}, \mathbf{w}_{3} \in G$, then under a CFL condition $\max \left\{\alpha_{f}^{1}, \alpha_{f}^{2}\right\} \lambda_{1} \leq 1$, where $\alpha_{f}^{1}$ is the parameter in the numerical flux $\widehat{\mathbf{f}}\left(\mathbf{w}_{1}, \mathbf{w}_{2}\right)$ and $\alpha_{f}^{2}$ is the one for $\widehat{\mathbf{f}}\left(\mathbf{w}_{2}, \mathbf{w}_{3}\right)$, then we have

$$
\mathbf{H}_{1}\left(\mathbf{w}_{1}, \mathbf{w}_{2}, \mathbf{w}_{3}, \lambda_{1}\right) \in G \text {. }
$$

Similarly, under the CFL condition $\max \left\{\alpha_{g}^{1}, \alpha_{g}^{2}\right\} \lambda_{2} \leq 1$ with $\alpha_{g}^{1}$ and $\alpha_{g}^{2}$ parameters for $\widehat{\mathbf{g}}\left(\mathbf{w}_{1}, \mathbf{w}_{2}\right)$ and $\widehat{\mathbf{g}}\left(\mathbf{w}_{2}, \mathbf{w}_{3}\right)$, respectively, then we have

$$
\mathbf{H}_{2}\left(\mathbf{w}_{1}, \mathbf{w}_{2}, \mathbf{w}_{3}, \lambda_{2}\right) \in G
$$

To continue, we use $L$-point Gauss quadratures with $L \geq k+1$ for accuracy to approximate the integrals in (3.1). More details of this requirement can be found in [4]. The Gauss quadrature points on $\left[x_{i-\frac{1}{2}}, x_{i+\frac{1}{2}}\right]$ and $\left[y_{j-\frac{1}{2}}, y_{j+\frac{1}{2}}\right]$ are denoted by

$$
p_{i}^{x}=\left\{x_{i}^{\beta}: \beta=1, \cdots, L\right\} \text { and } p_{j}^{y}=\left\{y_{j}^{\beta}: \beta=1, \cdots, L\right\}
$$

respectively. Also, we denote $w_{\beta}$ as the corresponding weights on the interval $\left[-\frac{1}{2}, \frac{1}{2}\right]$. Different from the notations in previous sections, we use

$$
\hat{p}_{i}^{x}=\left\{\hat{x}_{i}^{\alpha}: \alpha=0, \cdots, M\right\} \text { and } \hat{p}_{j}^{y}=\left\{\hat{y}_{j}^{\alpha}: \alpha=0, \cdots, M\right\}
$$

as the Gauss-Lobatto points on $\left[x_{i-\frac{1}{2}}, x_{i+\frac{1}{2}}\right]$ and $\left[y_{j-\frac{1}{2}}, y_{j+\frac{1}{2}}\right]$, respectively. Also, we denote $\hat{w}_{\alpha}$ as the corresponding weights on the interval $\left[-\frac{1}{2}, \frac{1}{2}\right]$.

Then the numerical scheme (3.1) becomes

$$
\begin{aligned}
\overline{\mathbf{w}}_{i j}^{n+1} & =\overline{\mathbf{w}}_{i j}^{n}+\lambda_{1} \sum_{\beta=1}^{L} w_{\beta}\left[\widehat{\mathbf{f}}\left(\mathbf{w}_{i-\frac{1}{2}, \beta}^{-}, \mathbf{w}_{i-\frac{1}{2}, \beta}^{+}\right)-\widehat{\mathbf{f}}\left(\mathbf{w}_{i+\frac{1}{2}, \beta}^{-}, \mathbf{w}_{i+\frac{1}{2}, \beta}^{+}\right)\right] \\
& +\lambda_{2} \sum_{\beta=1}^{L} w_{\beta}\left[\widehat{\mathbf{g}}\left(\mathbf{w}_{\beta, j-\frac{1}{2}}^{-}, \mathbf{w}_{\beta, j-\frac{1}{2}}^{+}\right)-\widehat{\mathbf{g}}\left(\mathbf{w}_{\beta, j+\frac{1}{2}}^{-}, \mathbf{w}_{\beta, j+\frac{1}{2}}^{+}\right)\right]
\end{aligned}
$$

where $\mathbf{w}_{i-\frac{1}{2}, \beta}^{-}=\mathbf{w}_{i-\frac{1}{2}, j}^{-}\left(y_{j}^{\beta}\right)$ is a point value in the Gauss quadrature. Likewise for the other point values. As the general treatment, we rewrite the cell average on the right hand side as

$$
\overline{\mathbf{w}}_{i j}^{n}=\sum_{\alpha=0}^{M} \sum_{\beta=1}^{L} \hat{w}_{\alpha} w_{\beta} \mathbf{w}_{\alpha \beta}^{1}=\sum_{\alpha=0}^{M} \sum_{\beta=1}^{L} \hat{w}_{\alpha} w_{\beta} \mathbf{w}_{\beta \alpha}^{2},
$$


where $\mathbf{w}_{\alpha \beta}^{1}$ and $\mathbf{w}_{\beta \alpha}^{2}$ denote $\mathbf{w}_{i j}^{n}\left(\hat{x}_{i}^{\alpha}, y_{j}^{\beta}\right)$ and $\mathbf{w}_{i j}^{n}\left(x_{i}^{\beta}, \hat{y}_{j}^{\alpha}\right)$, respectively.

For each vertical edge, use $\alpha_{f}^{i-\frac{1}{2}, \beta}$ to denote the parameter of the numerical flux $\widehat{\mathbf{f}}\left(\mathbf{w}_{i-\frac{1}{2}, \beta}^{-}, \mathbf{w}_{i-\frac{1}{2}, \beta}^{+}\right)$ and for the horizontal edge use $\alpha_{g}^{\beta, j-\frac{1}{2}}$ for the parameter of $\widehat{\mathbf{g}}\left(\mathbf{w}_{\beta, j-\frac{1}{2}}^{-}, \mathbf{w}_{\beta, j-\frac{1}{2}}^{+}\right)$. Then define

$$
\alpha_{f}^{i-\frac{1}{2}, j}=\max _{\beta=1, \cdots, L} \alpha_{f}^{i-\frac{1}{2}, \beta}, \quad \alpha_{g}^{i, j-\frac{1}{2}}=\max _{\beta=1, \cdots, L} \alpha_{g}^{\beta, j-\frac{1}{2}}
$$

Let $\mu=\max _{i, j} \alpha_{f}^{i-\frac{1}{2}, j} \lambda_{1}+\max _{i, j} \alpha_{g}^{i, j-\frac{1}{2}} \lambda_{2}$, then scheme (3.4) can be written as

$$
\begin{aligned}
\overline{\mathbf{w}}_{i j}^{n+1}=C_{1} \sum_{\beta=1}^{L} w_{\beta}\left(\sum_{\alpha=1}^{M-1} \hat{w}_{\alpha} \mathbf{w}_{\alpha \beta}^{1}+\hat{w}_{0} \mathbf{H}_{1}\left(\mathbf{w}_{i-\frac{1}{2}, \beta}^{-}, \mathbf{w}_{i-\frac{1}{2}, \beta}^{+}, \mathbf{w}_{i+\frac{1}{2}, \beta}^{-}, \frac{\lambda_{1}}{\hat{w}_{0} C_{1}}\right)\right. \\
\left.+\hat{w}_{M} \mathbf{H}_{1}\left(\mathbf{w}_{i-\frac{1}{2}, \beta}^{+}, \mathbf{w}_{i+\frac{1}{2}, \beta}^{-}, \mathbf{w}_{i+\frac{1}{2}, \beta}^{+}, \frac{\lambda_{1}}{\hat{w}_{M} C_{1}}\right)\right) \\
+C_{2} \sum_{\beta=1}^{L} w_{\beta}\left(\sum_{\alpha=1}^{M-1} \hat{w}_{\alpha} \mathbf{w}_{\beta \alpha}^{2}+\hat{w}_{0} \mathbf{H}_{2}\left(\mathbf{w}_{\beta, j-\frac{1}{2}}^{-}, \mathbf{w}_{\beta, j-\frac{1}{2}}^{+}, \mathbf{w}_{\beta, j+\frac{1}{2}}^{-}, \frac{\lambda_{2}}{\hat{w}_{0} C_{2}}\right)\right. \\
\left.+\hat{w}_{M} \mathbf{H}_{2}\left(\mathbf{w}_{\beta, i-\frac{1}{2}}^{+}, \mathbf{w}_{\beta, i+\frac{1}{2}}^{-}, \mathbf{w}_{\beta, j+\frac{1}{2}}^{+}, \frac{\lambda_{2}}{\hat{w}_{M} C_{2}}\right)\right),
\end{aligned}
$$

where

$$
C_{1}=\frac{\max _{i, j} \alpha_{f}^{i-\frac{1}{2}, j} \lambda_{1}}{\mu}, \quad C_{2}=\frac{\max _{i, j} \alpha_{g}^{i, j-\frac{1}{2}} \lambda_{2}}{\mu} .
$$

In (3.5), $\overline{\mathbf{w}}_{i j}^{n+1}$ is the convex combination of $\mathbf{w}, \mathbf{H}_{\mathbf{1}}$ and $\mathbf{H}_{\mathbf{2}}$. Therefore, we have the following theorem.

Theorem 3.1. Suppose $\mathbf{w}^{n} \in G$ in scheme (3.4), then $\overline{\mathbf{w}}^{n+1} \in G$, under the $C F L$ condition

$$
\frac{\Delta t}{\Delta x} \max _{i, j} \alpha_{f}^{i-\frac{1}{2}, j}+\frac{\Delta t}{\Delta y} \max _{i, j} \alpha_{g}^{i, j-\frac{1}{2}} \leq \hat{w}_{0}
$$

Remark 3.1. It is straightforward to obtain the bound that

$$
F_{1}(\mathbf{w}) \leq 1, \quad F_{2}(\mathbf{w}) \leq 1, \quad \forall \mathbf{w} \in G
$$

In practice, we can replace $\max _{i, j} \alpha_{f}^{i-\frac{1}{2}, j}$ and $\max _{i, j} \alpha_{g}^{i, j-\frac{1}{2}}$ by 1 to obtain the CFL condition.

Based on the above theorem, the numerical cell average we obtain is in $G$. Of course, the numerical solution $\mathbf{w}_{i j}^{n+1}$ might still be placed outside. Hence, we have to modify the 
numerical solution while keeping the cell average untouched. Due to the rounding error, we define

$$
\begin{gathered}
G^{\varepsilon}=\left\{\mathbf{w}=\left(\begin{array}{c}
D \\
m \\
n \\
E
\end{array}\right): D \geq \varepsilon, E \geq \sqrt{m^{2}+n^{2}+D^{2}+\varepsilon}\right\}, \\
\partial G^{\varepsilon}=\left\{\mathbf{w}=\left(\begin{array}{c}
D \\
m \\
n \\
E
\end{array}\right): D \geq \varepsilon, E=\sqrt{D^{2}+m^{2}+n^{2}+\varepsilon}\right\} .
\end{gathered}
$$

Then the modification of $\mathbf{w}_{i j}^{n}$ is given in the following steps.

- Set up a small number $\varepsilon=10^{-13}$.

- If $\bar{D}_{i j}^{n}>\varepsilon$, then proceed to the following steps. Otherwise, $D_{i j}^{n}$ is identified as the approximation to vacuum. We take $\widetilde{\mathbf{w}}_{i j}^{n}=\overline{\mathbf{w}}_{i j}^{n}$ as the numerical solution and skip the following steps.

- Modify the density: Compute $b_{i j}=\min _{\alpha \beta}\left\{D_{i j}^{n}\left(\widehat{x}_{i}^{\alpha}, y_{j}^{\beta}\right), D_{i j}^{n}\left(x_{i}^{\beta}, \widehat{y}_{j}^{\alpha}\right)\right\}$. If $b_{i j}<\varepsilon$, then take $\widetilde{D}_{i j}^{n}$ as

$$
\widetilde{D}_{i j}^{n}=\bar{D}_{i j}^{n}+\theta_{i j}^{D}\left(D_{i j}^{n}-\bar{D}_{i j}^{n}\right)
$$

with

$$
\theta_{i j}^{D}=\frac{\bar{D}_{i j}^{n}-\varepsilon}{\bar{D}_{i j}^{n}-b_{i j}},
$$

and use $\widetilde{D}_{i j}^{n}$ as the new numerical density $D_{i j}^{n}$.

- Enforce $E_{i j}^{n} \geq \sqrt{\left(m_{i j}^{n}\right)^{2}+\left(n_{i j}^{n}\right)^{2}+\left(D_{i j}^{n}\right)^{2}+\epsilon}$ in each cell $I_{i j}$ : Consider $\mathbf{w}_{\alpha \beta}^{1}$ and $\mathbf{w}_{\beta \alpha}^{2}$ in the cell $I_{i j}$, respectively. If $\mathbf{w}_{\alpha \beta}^{1} \in G^{\varepsilon}$, then take $\theta_{\alpha \beta}^{1}=1$. Otherwise, take $\theta_{\alpha \beta}^{1}$ to be the root of the equation

$$
\delta_{2}\left((1-t) \overline{\mathbf{w}}_{i j}^{n}+t \mathbf{w}_{\alpha \beta}^{1}\right)=0, \quad t \in(0,1) .
$$

where $\delta_{2}(\mathbf{w})=E-\sqrt{m^{2}+n^{2}+D^{2}+\varepsilon}$. Alternatively, the $t$ can be obtained in the same way as in Remark 2.3. Similarly, we can define $\theta_{\beta \alpha}^{2}$ in the same way for $\mathbf{w}_{\beta \alpha}^{2}$. 
Finally, we use

$$
\widetilde{\mathbf{w}}_{i j}^{n}=\overline{\mathbf{w}}_{i j}^{n}+\theta\left(\mathbf{w}_{i j}^{n}-\overline{\mathbf{w}}_{i j}^{n}\right), \quad \theta=\min _{\alpha, \beta}\left\{\theta_{\alpha \beta}^{1}, \theta_{\beta \alpha}^{2}\right\}
$$

as the DG approximation in cell $I_{i j}$.

Now we demonstrate the $L^{1}$-stability of the bound-preserving DG scheme. Following the same analysis as in section 2, we have

$$
\left\|D^{n}\right\|_{L^{1}}=\left\|D^{0}\right\|_{L^{1}} \quad \text { and } \quad\left\|E^{n}\right\|_{L^{1}}=\left\|E^{0}\right\|_{L^{1}}
$$

It is easy to check that

$$
m=\frac{u \Gamma}{\Gamma-\sigma} E \quad \text { and } \quad n=\frac{v \Gamma}{\Gamma-\sigma} E,
$$

where $\sigma=\frac{(\gamma-1)(h-1)}{h \gamma^{2}} \leq \Gamma-1$. Therefore,

$$
\|m\|_{L^{1}}+\|n\|_{L^{1}} \leq \frac{(|u|+|v|) \Gamma}{\Gamma-\sigma}\|E\|_{L^{1}} \leq \sqrt{2} \Gamma\|E\|_{L^{1}} .
$$

In the last inequality, we use the fact that $u^{2}+v^{2} \leq 1$.

The above analysis yields the $L^{1}$-stability of the scheme:

$$
\left\|\mathbf{w}^{n}\right\|_{L^{1}} \leq\left\|D^{n}\right\|_{L^{1}}+(\sqrt{2} \Gamma+1)\left\|E^{n}\right\|_{L^{1}}=\left\|D^{0}\right\|_{L^{1}}+(\sqrt{2} \Gamma+1)\left\|E^{0}\right\|_{L^{1}} \leq(\sqrt{2} \Gamma+1)\left\|\mathbf{w}^{0}\right\|_{L^{1}},
$$

where $\|\mathbf{w}\|_{L^{1}}=\|D\|_{L^{1}}+\|m\|_{L^{1}}+\|n\|_{L^{1}}+\|E\|_{L^{1}}$.

\section{Application to relativistic jets}

In this section, we study the relativistic axisymmetric jets which is described in the twodimensional cylindrical coordinates $(r, z)$. We adopt the governing system in [23] and multiply both sides of the system by $r$. Then it reads

$$
\mathbf{w}_{t}+\mathbf{f}(\mathbf{w})_{r}+\mathbf{g}(\mathbf{w})_{z}=\mathbf{s}(r, \mathbf{w})
$$

with

$$
\mathbf{w}=\left(\begin{array}{c}
r D \\
r m \\
r n \\
r E
\end{array}\right), \mathbf{f}(\mathbf{w})=\left(\begin{array}{c}
r D u \\
r m u+r p \\
r n u \\
r m
\end{array}\right), \mathbf{g}(\mathbf{w})=\left(\begin{array}{c}
r D v \\
r m v \\
r n v+r p \\
r n
\end{array}\right), \mathbf{s}=\left(\begin{array}{c}
0 \\
p \\
0 \\
0
\end{array}\right) .
$$


We would like to mention that this formulation is not unique. We could also combine the $p$ term in the source and the $r p$ term in the momentum flux to form a non-conservative $r p_{r}$ term, which is closer to the underlying physics. However, since our method is based on the conservative form of the equation we will stay with (4.1).

Different from what we have discussed in section 3 , the source term $\mathbf{s}(r, \mathbf{w})$ is not zero. Therefore, we will demonstrate how to discretize the source terms. The techniques for the discretization of the flux terms and the modification of the numerical approximations follow from the same lines as discussed in section 3, and we will omit them.

In this section, the cell is defined as $I_{i j}=\left[r_{i-\frac{1}{2}}, r_{i+\frac{1}{2}}\right] \times\left[z_{j-\frac{1}{2}}, z_{j+\frac{1}{2}}\right]$, with $1 \leq i \leq N_{r}$, $1 \leq j \leq N_{z}$ and the mesh sizes in $r$ and $z$ directions are denoted as $\Delta r$ and $\Delta z$, respectively. If not otherwise stated in this section, the notations follow those in section 3. We consider high order schemes only, the one satisfied by the cell averages can be written as

$$
\begin{aligned}
\overline{\mathbf{w}}_{i j}^{n+1} & =\frac{1}{2} \overline{\mathbf{w}}_{i j}^{n}+\frac{\Delta t}{\Delta r \Delta z} \int_{z_{j-\frac{1}{2}}}^{z_{j+\frac{1}{2}}} \widehat{\mathbf{f}}\left(\mathbf{w}_{i-\frac{1}{2}, j}^{-}(z), \mathbf{w}_{i-\frac{1}{2}, j}^{+}(z)\right)-\widehat{\mathbf{f}}\left(\mathbf{w}_{i+\frac{1}{2}, j}^{-}(z), \mathbf{w}_{i+\frac{1}{2}, j}^{+}(z)\right) d z \\
& +\frac{\Delta t}{\Delta r \Delta z} \int_{r_{i-\frac{1}{2}}}^{r_{i+\frac{1}{2}}} \widehat{\mathbf{g}}\left(\mathbf{w}_{i, j-\frac{1}{2}}^{-}(r), \mathbf{w}_{i, j-\frac{1}{2}}^{+}(r)\right)-\widehat{\mathbf{g}}\left(\mathbf{w}_{i, j+\frac{1}{2}}^{-}(r), \mathbf{w}_{i, j+\frac{1}{2}}^{+}(r)\right) d r \\
& +\frac{1}{2} \overline{\mathbf{w}}_{i j}^{n}+\Delta t \overline{\mathbf{s}}
\end{aligned}
$$

where $\overline{\mathbf{s}}$ is the cell average of $\mathbf{s}$. We use $L$-point Gauss quadratures with $L \geq \frac{k+1}{2}$ to approximate $\overline{\mathbf{s}}$. The Gauss quadrature points on $\left[r_{i-\frac{1}{2}}, r_{i+\frac{1}{2}}\right]$ and $\left[z_{j-\frac{1}{2}}, z_{j+\frac{1}{2}}\right]$ are denoted by

$$
p_{i}^{r}=\left\{r_{i}^{\beta}: \beta=1, \cdots, L\right\} \text { and } p_{j}^{z}=\left\{z_{j}^{\beta}: \beta=1, \cdots, L\right\}
$$

respectively. Also, we denote $w_{\beta}$ as the corresponding weights on the interval $\left[-\frac{1}{2}, \frac{1}{2}\right]$. Then

$$
\frac{1}{2} \overline{\mathbf{w}}+\Delta t \overline{\mathbf{s}}=\sum_{\alpha=1}^{L} \sum_{\beta=1}^{L} w_{\alpha} w_{\beta} \mathbf{H}_{s}\left(\mathbf{w}_{i j}^{\alpha \beta}, \mathbf{s}_{i j}^{\alpha \beta}, \Delta t\right),
$$

where $\mathbf{w}_{i j}^{\alpha \beta}=\mathbf{w}_{i j}\left(r_{i}^{\alpha}, z_{j}^{\beta}\right), \mathbf{s}_{i j}^{\alpha \beta}=\mathbf{s}_{i j}\left(r_{i}^{\alpha}, \mathbf{w}_{i j}^{\alpha \beta}\right)$ and $\mathbf{H}_{s}(\mathbf{w}, \mathbf{s}, \Delta t)=\frac{1}{2} \mathbf{w}+\Delta t \mathbf{s}$. Since $\mathbf{s}$ is a function of $\mathbf{w}$ and $\mathrm{r}, \mathbf{H}_{s}$ can be written as a function of $\mathbf{w}, r$ and $\Delta t$, i.e. $\widetilde{\mathbf{H}}_{s}(\mathbf{w}, r, \Delta t)=$ $\mathbf{H}_{s}(\mathbf{w}, \mathbf{s}, \Delta t)$. We can choose $\Delta t$ sufficiently small, such that $\widetilde{\mathbf{H}}_{s}(\mathbf{w}, r, \Delta t)=\mathbf{H}_{s}(\mathbf{w}, \mathbf{s}, \Delta t) \in$ $G$, and the result is given in the following lemma, whose proof is given in Appendix B. 
Lemma 4.1. Suppose $\mathbf{w} \in G$, if the time mesh size $\Delta t$ satisfies

$$
\Delta t \leq \frac{r}{2} \widetilde{\alpha}(\mathbf{w}, r)
$$

where

$$
\widetilde{\alpha}(\mathbf{w}, r)=\frac{\sqrt{\left(u \Gamma h \gamma^{2}\right)^{2}-(\Gamma-1)^{2}(h-1)^{2}-\Gamma^{2} \gamma^{2}(h-1)^{2}+2 \Gamma h \gamma^{2}(h-1)}-u h \Gamma \gamma^{2}}{(\Gamma-1)(h-1)},
$$

then $\widetilde{\mathbf{H}}_{s}(\mathbf{w}, r, \Delta t) \in G$.

With the above lemma, we can state the following theorem

Theorem 4.1. Suppose $\mathbf{w}^{n} \in G$, then $\overline{\mathbf{w}}^{n+1} \in G$ under the conditions

$$
\begin{gathered}
\Delta t \leq \max _{\substack{1 \leq \alpha, \beta \leq L \\
1 \leq i \leq N_{r} \\
1 \leq j \leq N_{z}}} \frac{r_{i}^{\alpha}}{2} \widetilde{\alpha}\left(\mathbf{w}_{i j}^{\alpha \beta}, r_{i}^{\alpha}\right) \\
\\
1 \leq
\end{gathered}
$$

and

$$
\frac{\Delta t}{\Delta r} \max _{i, j} \alpha_{f}^{i-\frac{1}{2}, j}+\frac{\Delta t}{\Delta z} \max _{i, j} \alpha_{g}^{i, j-\frac{1}{2}} \leq \frac{\hat{w}_{0}}{2}
$$

where $\alpha_{f}^{i-\frac{1}{2}, j}$ and $\alpha_{g}^{i, j-\frac{1}{2}}$ have the same definition as those in (3.6).

Now we have finished all the theoretical analysis.

\section{Numerical experiments}

In this section, we present numerical examples in both one and two dimensions to verify the bound-preserving property of the proposed method. In order to demonstrate the effectiveness of the proposed BP limiter, we have deliberately not applied any other non-oscillatory limiters, such as the TVD/TVB or WENO limiters. As expected from the theory, the numerical solution stays in the physical bounds and computations could proceed stably, even though there are spurious oscillations in some test results due to the lack of non-oscillatory limiters. We should emphasize that this bound-preserving limiter, which is extremely local (implemented completely inside each cell without using information from neighboring cells) 
and inexpensive, is not meant to substitute other non-oscillatory limiters or techniques. It can be used together with other non-oscillatory limiters or techniques, such as TVD/TVB or WENO limiters, or even WENO finite volume schemes, to obtain both bound-preserving and non-oscillatory performance, but we will not pursue such approach in this paper in order to be focused on the bound-preserving property. We would also like to emphasize that many of the examples are chosen to contain solutions particularly challenging in terms of the bound-preserving requirement, such that even with the usual TVD/TVB or WENO limiters added, the code would still fail without the proposed bound-preserving technique, due to the breaking of the physical bound which causes ill-posedness of the problem as well as the nonexistence of the solution to (1.9). Unless otherwise indicated, we consider the third-order RKDG method $(k=2)$ with the local Lax-Friedrichs numerical flux (2.3). The CFL number is set to be 0.15 . The specific heat ratio $\Gamma$ is taken to be $5 / 3$, unless otherwise stated.

\subsection{One-dimensional experiments}

Example 5.1.1 (Smooth flow). Consider a one-dimensional flow in the domain $\Omega=[0,1]$ with the following initial states

$$
\rho_{0}(x)=1+0.9999999 \sin (2 \pi x), \quad u_{0}(x)=0.9, \quad p_{0}(x)=1.0
$$

The boundary condition is set to be periodic. The exact solution is

$$
\rho(x, t)=1+0.9999999 \sin (2 \pi(x-0.9 t)), \quad u(x, t)=0.9, \quad p(x, t)=1.0
$$

In Table 5.1, we present the numerical results for the proposed method with and without the bound-preserving limiter. For the case $k=3$, we take $\Delta t=\operatorname{CFL}(\Delta x)^{4 / 3}$ so that the time error will not dominate. Since this is a high speed smooth flow with its lowest density near zero, the bound-preserving limiter does get turned on. As observed and discussed in [59] and [51], the SSP Runge-Kutta (RK) methods might degenerate the accuracy when the bound-preserving limiter is applied, while for the SSP multi-step (M-S) method, the full order of accuracy is recovered. 


\begin{tabular}{c|c|c|c|c|c|c|c}
\hline & & \multicolumn{2}{|c|}{ no limiter } & \multicolumn{2}{|c|}{ with limiter (RK) } & \multicolumn{2}{c}{ with limiter (M-S) } \\
\hline$k$ & $h$ & $L^{2}$ error & order & $L^{2}$ error & order & $L^{2}$ error & order \\
\hline \multirow{6}{*}{1} & $1 / 20$ & 3.50 e-2 & - & 4.85 e-2 & - & 4.55 e-2 & - \\
& $1 / 40$ & 8.72 e-3 & 2.00 & 1.06 e-2 & 2.11 & 1.05 e-2 & 2.12 \\
& $1 / 80$ & 2.17 e-3 & 2.01 & 2.55 e-3 & 2.05 & 2.55 e-3 & 2.03 \\
& $1 / 160$ & 5.43 e-4 & 2.00 & 6.13 e-4 & 2.03 & 6.11 e-4 & 2.06 \\
& $1 / 320$ & 1.36 e-4 & 2.00 & 1.50 e-4 & 2.04 & 1.49 e-4 & 2.03 \\
\hline \multirow{6}{*}{2} & $1 / 20$ & 2.16 e-3 & - & 3.47 e-3 & - & 2.40 e-3 & - \\
& $1 / 40$ & 2.77 e-4 & 2.97 & 5.05 e-4 & 2.78 & 2.79 e-4 & 3.10 \\
& $1 / 80$ & 3.48 e-5 & 2.99 & 9.42 e-5 & 2.42 & 3.48 e-5 & 3.00 \\
& $1 / 160$ & 4.35 e-6 & 3.00 & 1.87 e-5 & 2.33 & 4.35 e-6 & 3.00 \\
& $1 / 320$ & 5.44 e-7 & 3.00 & 3.68 e-6 & 2.35 & 5.44 e-7 & 3.00 \\
\hline \multirow{6}{*}{3} & $1 / 20$ & 8.99 e-5 & - & 1.82 e-4 & - & 1.52 e-4 & - \\
& $1 / 40$ & 5.60 e-6 & 4.00 & 2.09 e-5 & 3.12 & 5.82 e-6 & 8.03 \\
& $1 / 80$ & 3.46 e-7 & 4.02 & 2.30 e-6 & 3.18 & 3.50 e-7 & 4.05 \\
& $1 / 160$ & 2.16 e-8 & 4.00 & 2.70 e-7 & 3.09 & 2.19 e-8 & 4.00 \\
& $1 / 320$ & 1.35 e-9 & 4.00 & 1.86 e-8 & 3.86 & 1.46 e-9 & 3.91 \\
\hline
\end{tabular}

Table 5.1: Example 5.1.1: One-dimensional accuracy test at $T=0.4$ for the second-, thirdand fourth-order DG methods with and without the BP limiters. The CFL number for the multistep method is one-third of that for the RK method. $k$ is the degree of polynomial and $h$ is the meshsize.

Moreover, in Figure 5.1, we plot the CPU time against the $L^{2}$ error for polynomial degrees $k=1,2,3$ with and without the BP limiter. The following two observations could be made: (i) As one of the advantages of high order DG methods, for a given error, the computational time spent is diminishing with increasing order; (ii) The additional cost introduced by the BP limiter is very small.

Example 5.1.2 (Moderate blast wave). The relativistic blast wave problems are standard tests (see [22]) for a numerical relativistic hydrodynamical code. The computational domain is $\Omega=[0,1]$. We first consider a moderate case, which has the initial states

$$
\left(\rho_{0}, u_{0}, p_{0}\right)= \begin{cases}(10.0,0.0,13.33), & x<0.5 \\ (1.0,0.0,0.0), & x>0.5\end{cases}
$$

The initial discontinuity gives rise to a transonic rarefaction wave propagating left, a shock wave propagating right and a contact discontinuity in between. The numerical approximation in Figure 5.2 shows that our method can resolve these structures quite well. Small oscillations are observed around the contact discontinuity, since we have not applied any non-oscillatory 


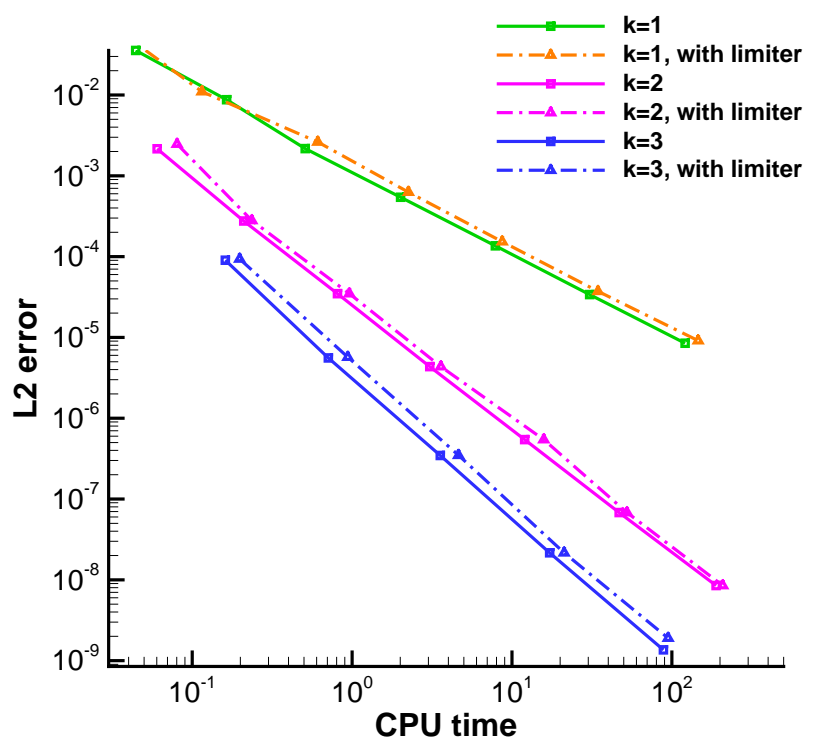

Figure 5.1: Example 5.1.1: Log-log plot of the CPU time against the $L^{2}$ error for polynomial degrees $k=1,2,3$ with and without the BP limiter.

limiters such as the TVD/TVB or WENO limiters. For the same reason, in some of the following examples, we also observe spurious oscillations due to the lack of non-oscillatory limiters.

Example 5.1.3 (Strong relativistic blast wave). Next we examine a much more challenging blast wave example, of which the initial states are

$$
\left(\rho_{0}, u_{0}, p_{0}\right)= \begin{cases}\left(1.0,0.0,10^{3}\right), & x<0.5 \\ (1.0,0.0,0.0), & x>0.5\end{cases}
$$

This example is more relativistic than the previous one. The high relativity is due to the large enthalpy of the left state, which is $h \simeq 2.5 \times 10^{3} \gg 1$. This results in a thermodynamically relativistic configuration. The structure of the solution is the same as the moderate case, except for the formation of a very thin dense shell behind the shock in the density and a highly curved profile for the rarefaction fan in the velocity. The relativistic shock propagating at a Lorentz factor $\gamma \simeq 6$ [54]. In Figure 5.3, despite of small oscillations, we can see that our method resolves the curved part in the profile of the velocity very well and captures the thin shell in the density with little smearing. 


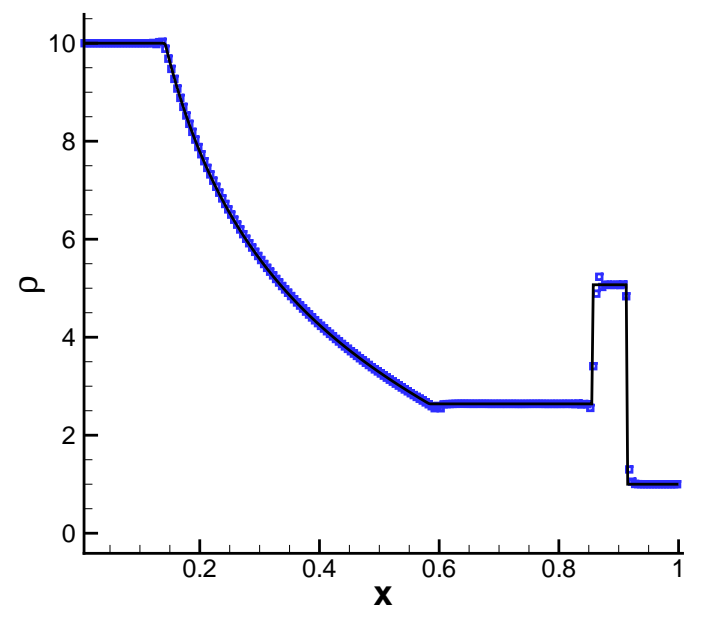

(a) density $\rho$

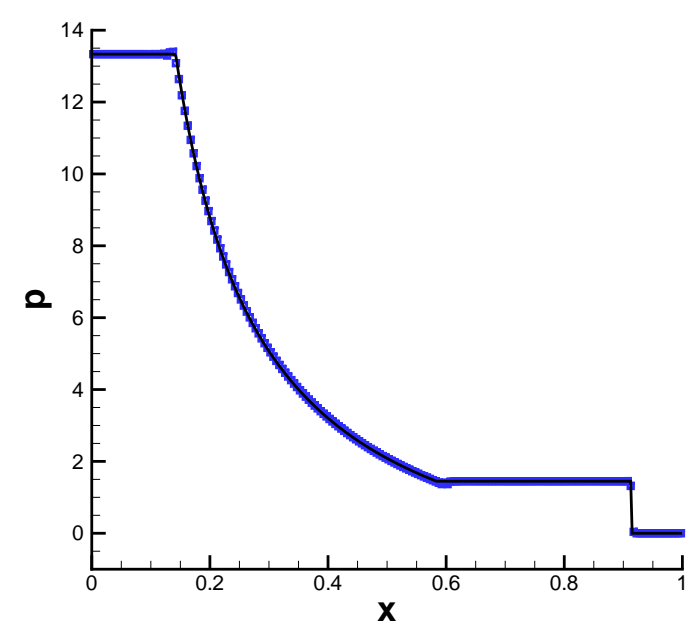

(b) pressure $p$

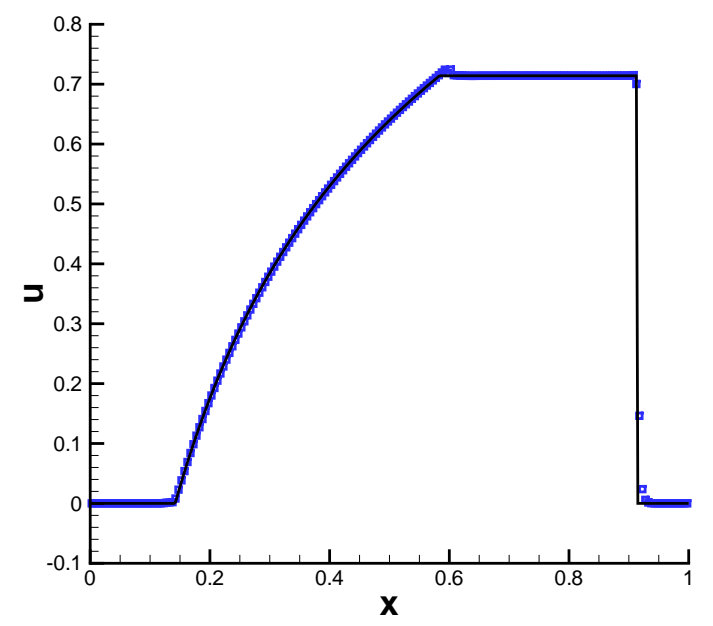

(c) velocity $u$

Figure 5.2: Example 5.1.2: Blast wave with initial condition (5.1) at $T=0.5$ on the mesh of 200 cells, approximated by the third-order RKDG method with the BP limiter. The squares represent the approximate cell averages and the solid line is the exact solution.

To further test the bound preserving property of our method, we consider a more extreme example. The initial condition now is

$$
\left(\rho_{0}, u_{0}, p_{0}\right)= \begin{cases}\left(1.0,0.0,10^{4}\right), & x<0.5 \\ (1.0,0.0,0.0), & x>0.5\end{cases}
$$

with the specific heat ratio $\Gamma=4 / 3$. In this case the enthalpy of the left state is $h \simeq 4 \times 10^{4}$. In the profile of the density, the width of the thin shell is approximately $2.5 \times 10^{-3}$ and the 


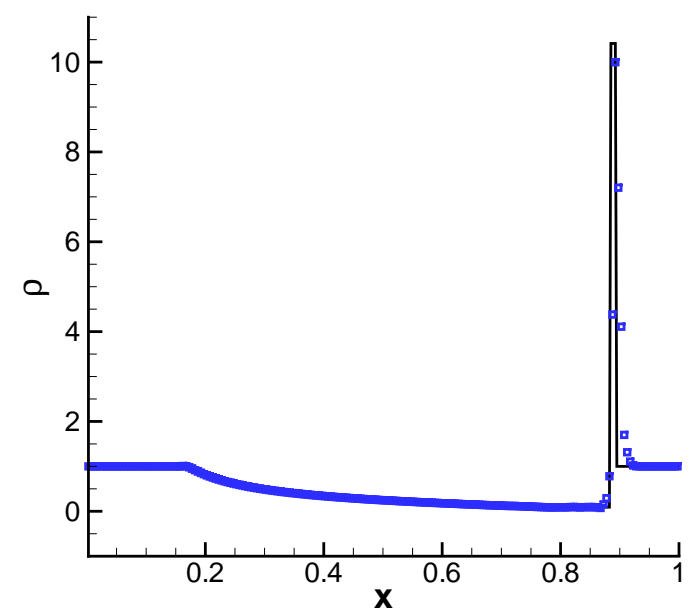

(a) density $\rho$

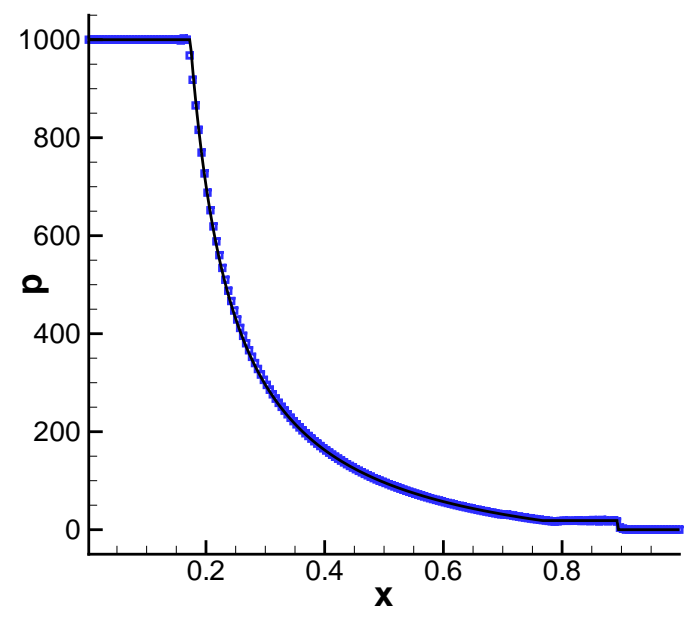

(b) pressure $p$

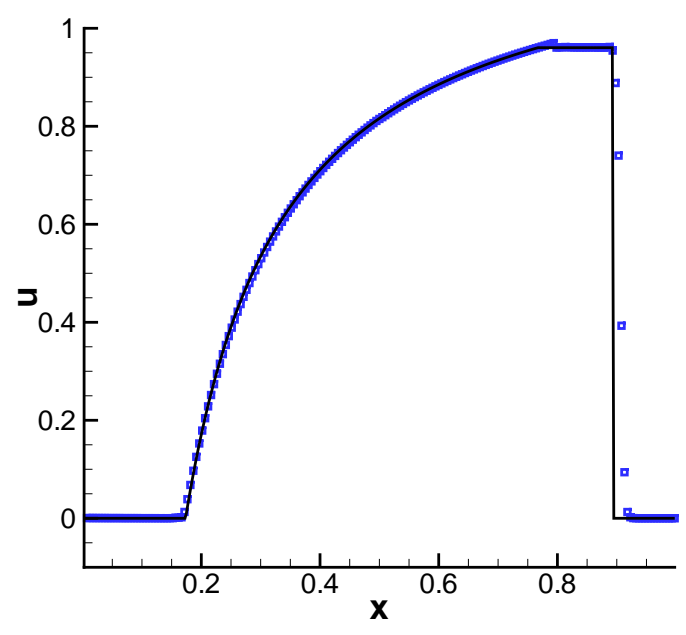

(c) velocity $u$

Figure 5.3: Example 5.1.3: Strong blast wave with initial condition (5.2) at $T=0.4$ approximated by the third-order RKDG method with the BP limiter on the mesh with 200 cells. The square represents the approximate cell averages and the solid line is the exact solution.

rarefaction part in the velocity becomes even more curved. In Figure 5.4, we can see the good performance of our proposed method.

Example 5.1.4 (Shock-heating problem). The shock-heating problem (see [7, 54, 21, 22]) is a standard benchmark problem to examine the ability of the method to deal with strong shocks. A warm wall is located at $x=0$ and cold gas flows in at $x=1.0$. When the gas 


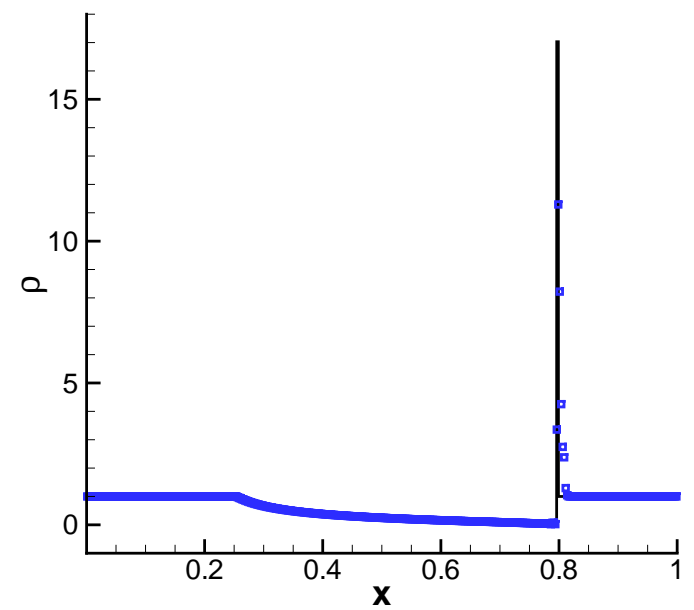

(a) density $\rho, 400$ cells

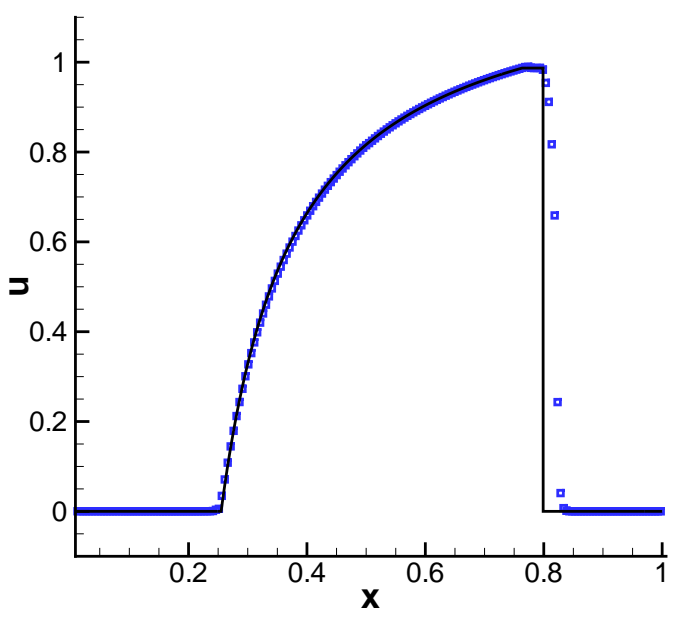

(c) velocity $u, 400$ cells

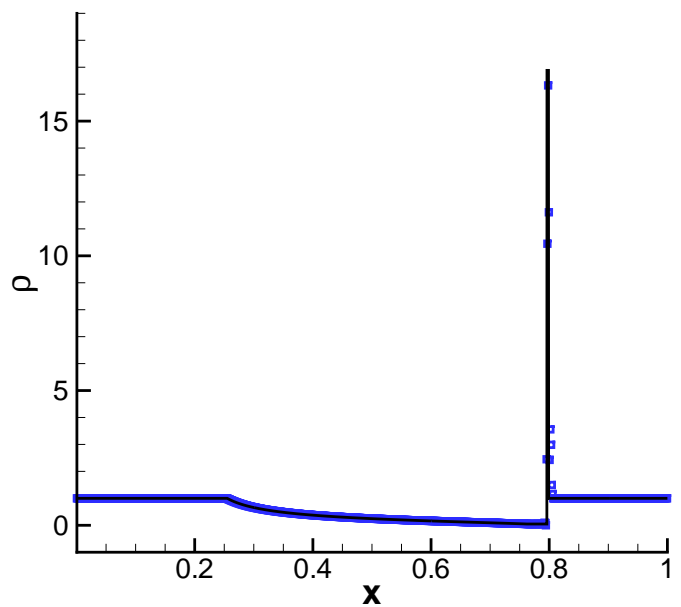

(b) density $\rho, 800$ cells

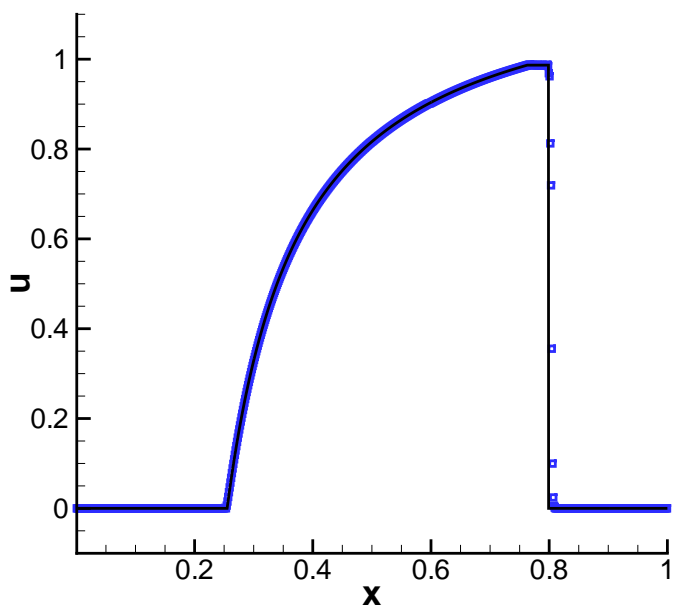

(d) velocity $u, 800$ cells

Figure 5.4: Example 5.1.3: Strong blast wave with initial condition (5.3) at $T=0.3$ approximated by the third-order RKDG method with the BP limiter. The mesh is decomposed into 400 cells (left column) and 800 cells (right column). The square represents the approximate cell averages and the solid line is the exact solution.

gets reflected on the wall, a reverse strong shock forms and propagates to the right. In our experiment, the inflow velocity is set to be $v_{i n}=0.999999$, with the corresponding Lorentz factor $\gamma=707.1$. The initial density is $\rho_{0}=1.0$ and the initial pressure $p_{0}=0.0$. The specific heat ratio in this example is $\Gamma=4 / 3$. The analytical solution can be found in [21].

In Figure 5.5, we observe that, due to the BP limiter, near the physical bounds, there is 
no oscillation and the bounds are well preserved. The shocks are sharply captured and the oscillations behind the shock may be eliminated with the non-oscillatory techniques such as the TVB or the WENO limiter. There is an undershoot in the left end point of the density, which is the so-called "wall-heating" effect. All these show the effectiveness of the proposed BP limiter in the ultra-relativistic regime with very strong shocks.

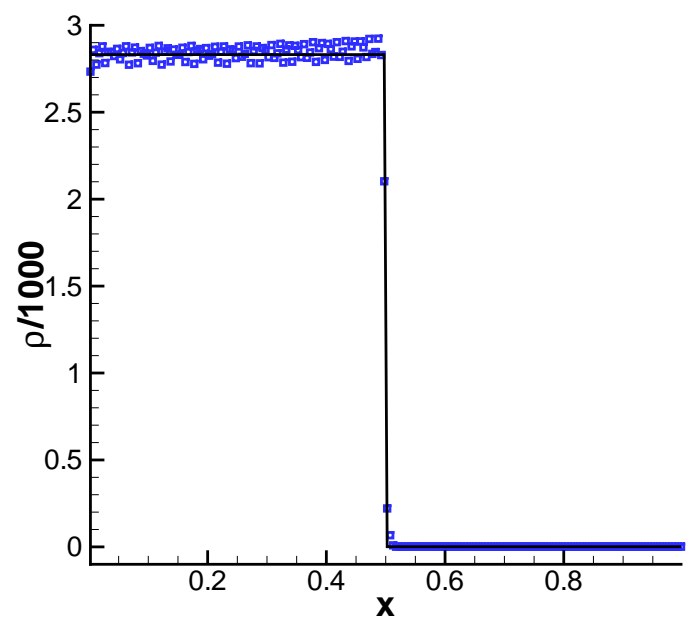

(a) density $\rho$

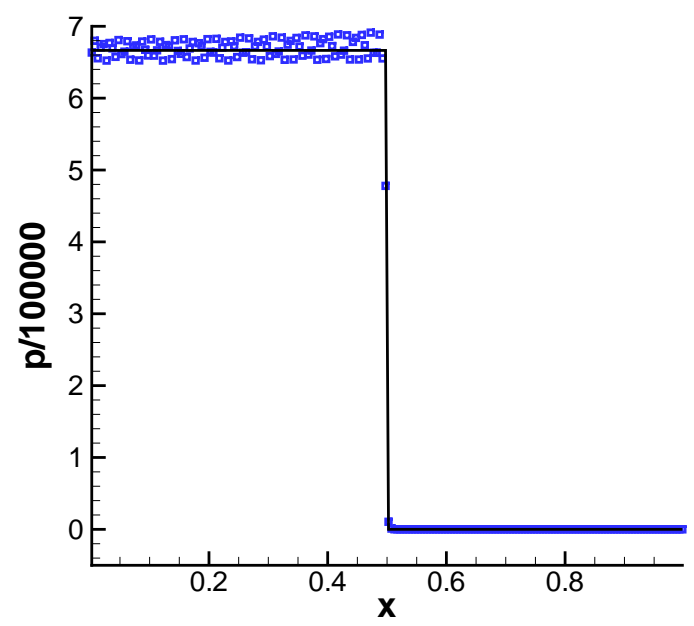

(b) pressure $p$

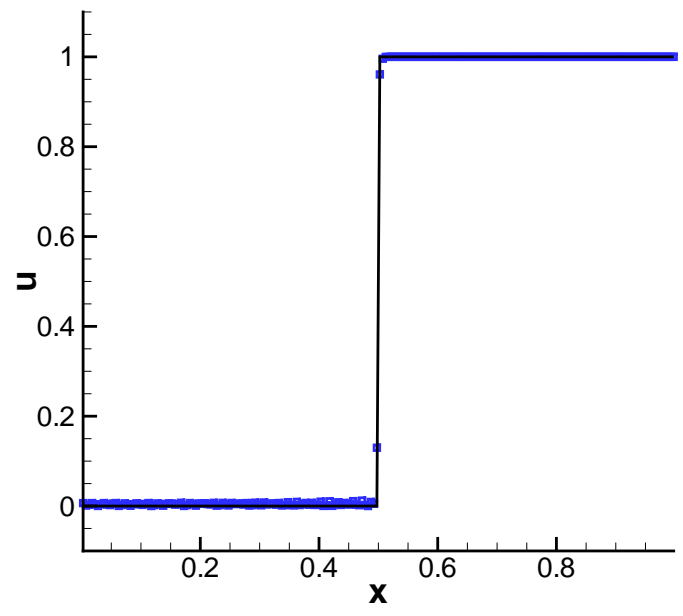

(c) velocity $u$

Figure 5.5: Example 5.1.4: One-dimensional shock-heating problem on the mesh of 200 cells, at $T=1.5$ with $v_{i n}=0.999999$, approximated by the third-order RKDG method with the BP limiter. The squares represent the numerical results and the solid line is the exact solution. 
Example 5.1.5 (One-dimensional Riemann problem with non-zero transverse velocity). The last one-dimensional test is the Riemann problem with non-zero transverse velocity, see [54] and [25]. The domain is still $\Omega=[0,1]$ and the initial states are

$$
\left(\rho_{0}, u_{0}, v_{0}, p_{0}\right)= \begin{cases}\left(1.0,0.0,0.9,10^{3}\right), & x<0.5 \\ \left(1.0,0.0,0.9,10^{-2}\right), & x>0.5\end{cases}
$$

Compared with Example 5.1.3, where there is no transverse velocity, the density has a smaller jump and a wider dense shell, however the distance between the tail of the rarefaction and the contact discontinuity is much smaller, which requires very high resolution to resolve the structure. The purpose of this example is to show the robustness of the BP limiter even when the transverse speed is nonzero and close to the speed of light. Admittedly, to correctly restore the right wave speed, it is unavoidable to refine the mesh (see the comparison between 400 cells and 6400 cells in Figures 5.6 and 5.7 and also results in [54] with the adaptive mesh refinement technique), but this goes beyond the purpose of this paper and we will not pursue it here. Our results on meshes of 400 and 6400 cells are presented in Figure 5.6 and Figure 5.7. These results match those in [54] and [25] well.

We further test this problem with a transverse velocity $v=0.999$. In this case, the dense shell in the density is much thinner and the transverse velocity increases from $v=0.999$ to $v=0.99967$ at the rarefaction part, which corresponds to a Lorentz factor $\gamma \simeq 39$. We see in Figure 5.8, our method is still robust in this severe case.

\subsection{Two-dimensional experiments}

Example 5.2.1 (Smooth flow). First, the accuracy of the method in 2-D is checked by considering the following smooth flow in the domain $\Omega=[0, \sqrt{2}]^{2}$.

$$
\rho=1+0.999999 \sin [2 \pi(\mathbf{r}-\mathbf{v} t) \cdot \mathbf{b}], \quad v_{x}=0.9, \quad v_{y}=0.2, \quad p=1.0
$$

where $\mathbf{r}=(x, y), \mathbf{v}=\left(v_{x}, v_{y}\right)$ and $\mathbf{b}=(\cos \alpha, \sin \alpha)$ is the direction vector, along which the wave propagates. In this example we take $\alpha=\pi / 4$. In Table 5.2, the numerical errors in 


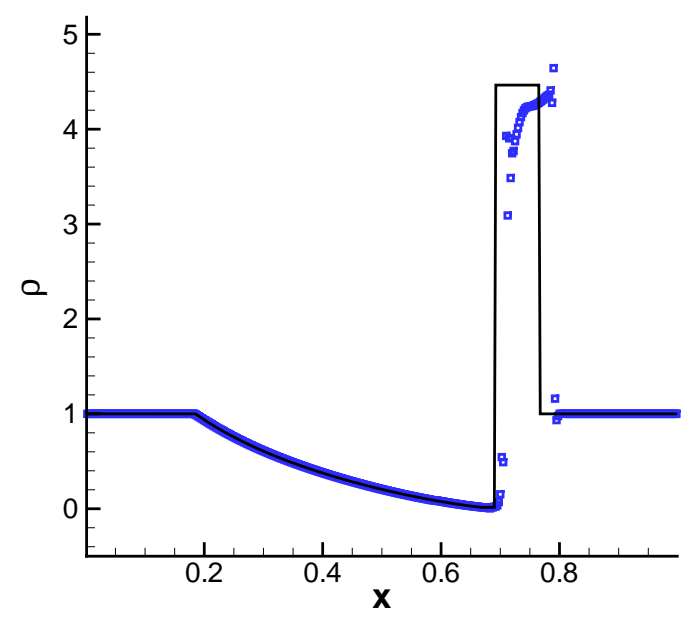

(a) density $\rho, 400$ cells

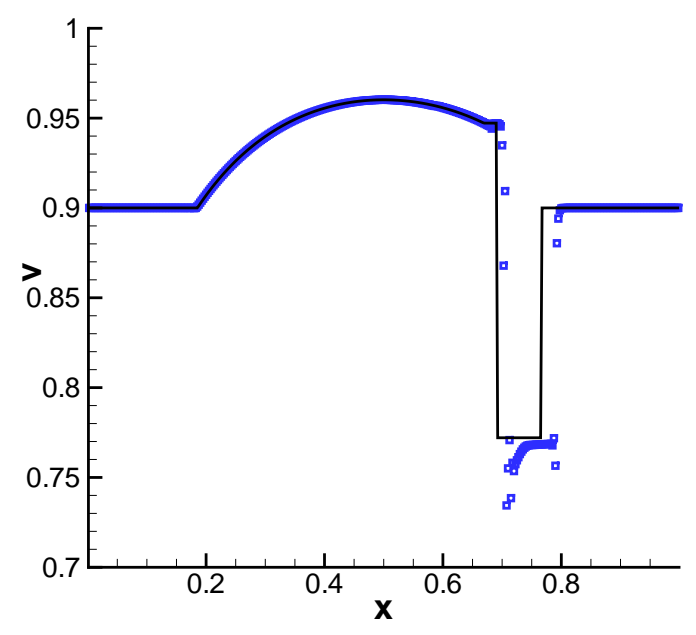

(c) velocity $v, 400$ cells

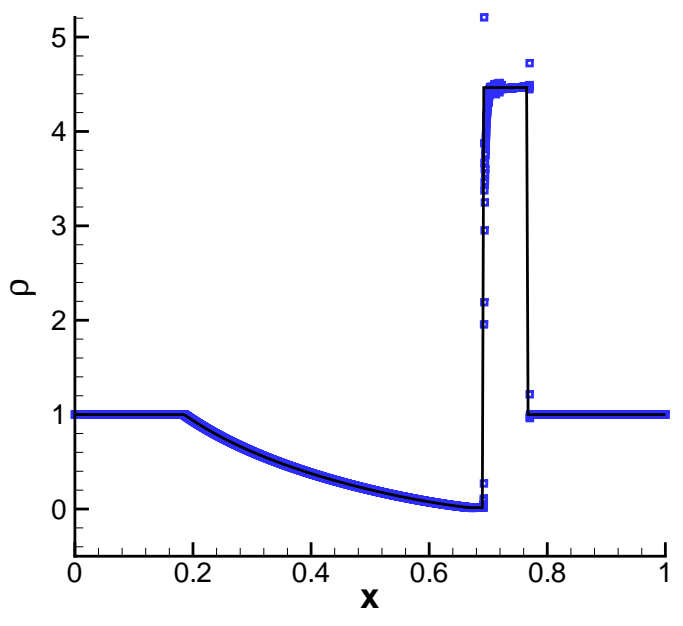

(b) density $\rho, 6400$ cells

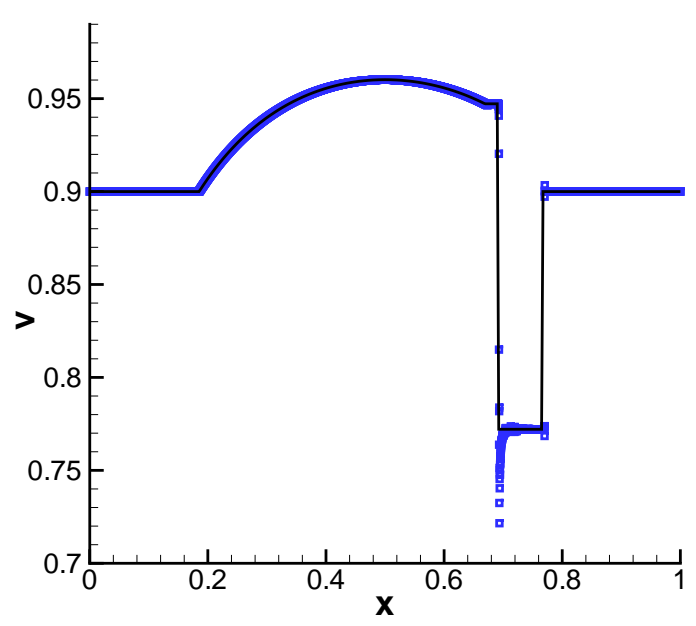

(d) velocity $v, 6400$ cells

Figure 5.6: Example 5.1.5: One-dimensional Riemann problem with non-zero transverse velocity at $T=0.6$, approximated by the third-order RKDG method with the BP limiter. Approximations of the density and the transverse velocity on meshes of 400 and 6400 cells. The squares represent the approximate cell averages and the solid lines are the exact solutions.

$L^{2}$ and $L^{\infty}$ norms of the RKDG method with the BP limiter are listed. We can see that, in this case, the limiter preserves the high-order accuracy.

Example 5.2.2 (Oblique shock wave). Next, let us check a two-dimensional oblique 1D shock tube test in the domain $[0, \sqrt{2} / 2]^{2}$. We consider the strong blast wave in Example 


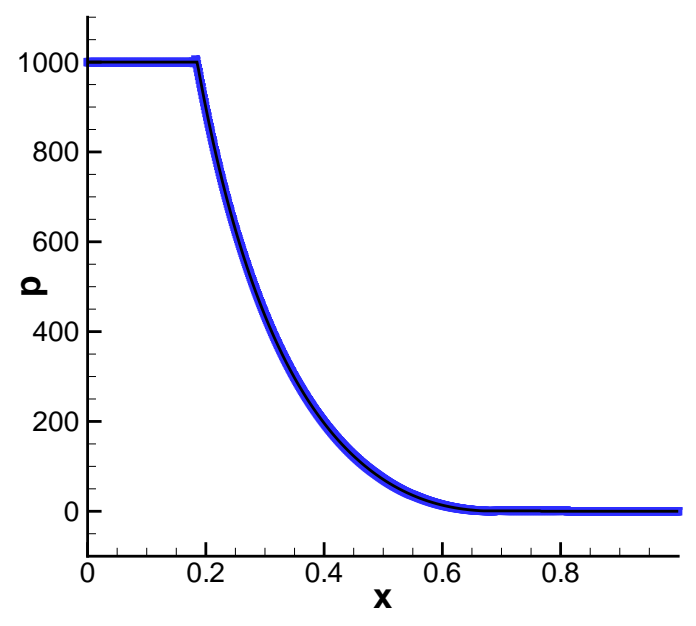

(a) pressure $p, 400$ cells

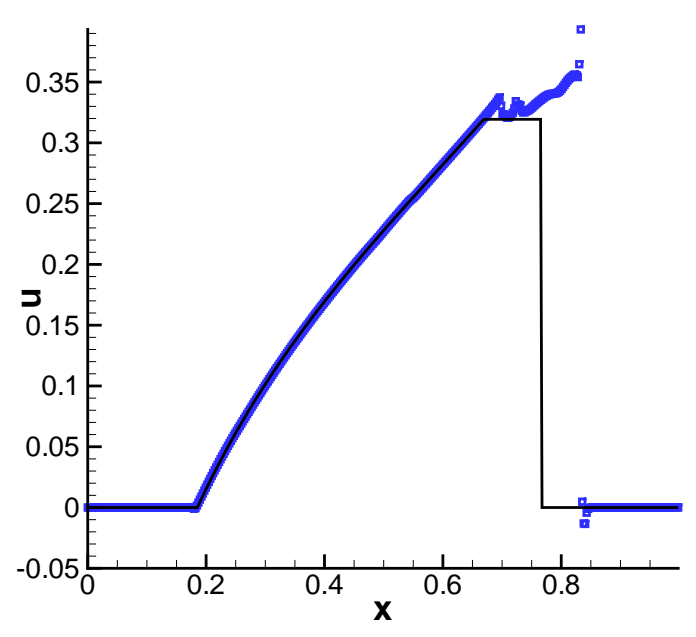

(c) velocity $u, 400$ cells

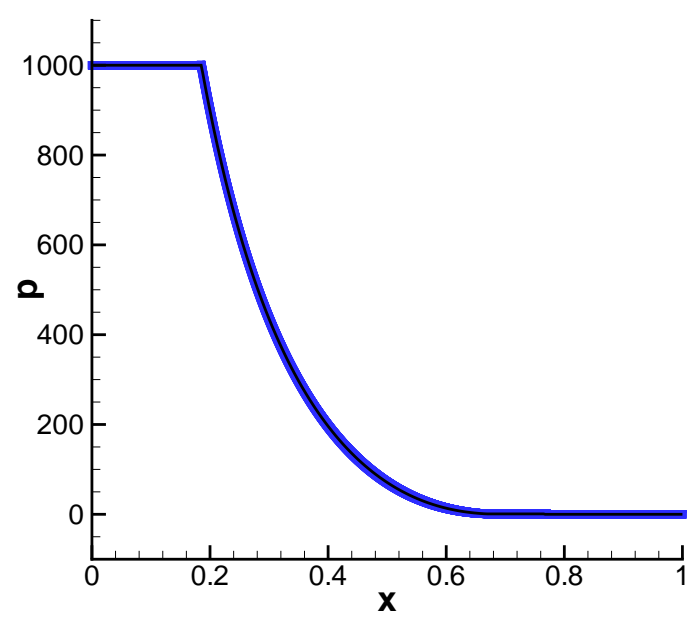

(b) pressure $p, 6400$ cells

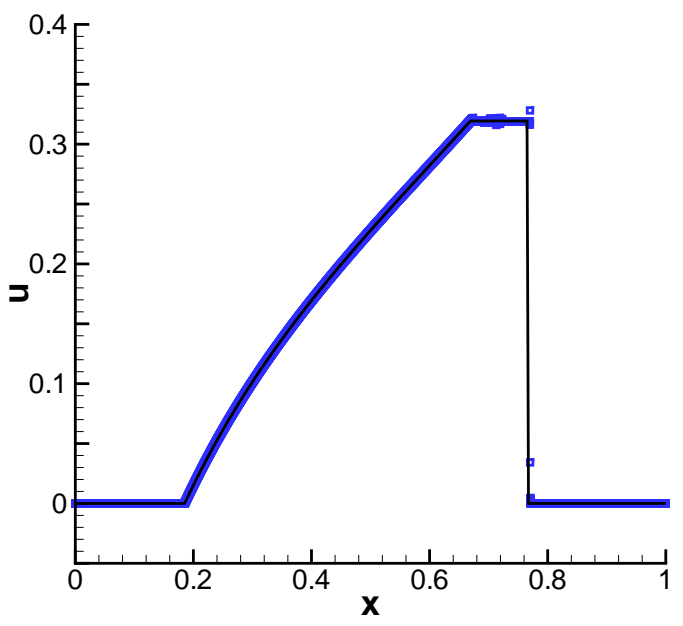

(d) velocity $u, 6400$ cells

Figure 5.7: Example 5.1.5: One-dimensional Riemann problem with non-zero transverse velocity at $T=0.6$, approximated by the third-order RKDG method with the BP limiter. The approximations of $u$ and $p$ on meshes of 400 and 6400 cells are presented respectively. The squares represent the approximate cell averages and the solid lines are the exact solutions.

5.1.3 propagating along the direction at a 45 degree angle. Initially, the state is divided into two parts by the line $x+y=1$ and is set to be

$$
\left(\rho_{0}, u_{0}, v_{0}, p_{0}\right)=\left\{\begin{array}{ll}
\left(1.0,0.0,0.0,10^{3}\right), & x+y<1 \\
(1.0,0.0,0.0,0.0), & x+y>1
\end{array} .\right.
$$

The domain is divided into $120 \times 120$ cells and the terminal time $T=0.3$. In Figure 5.9, we 


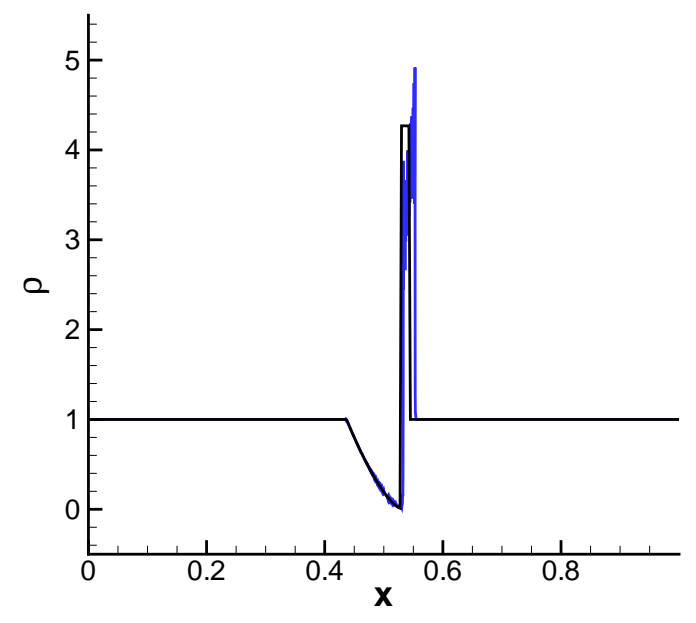

(a) density $\rho$

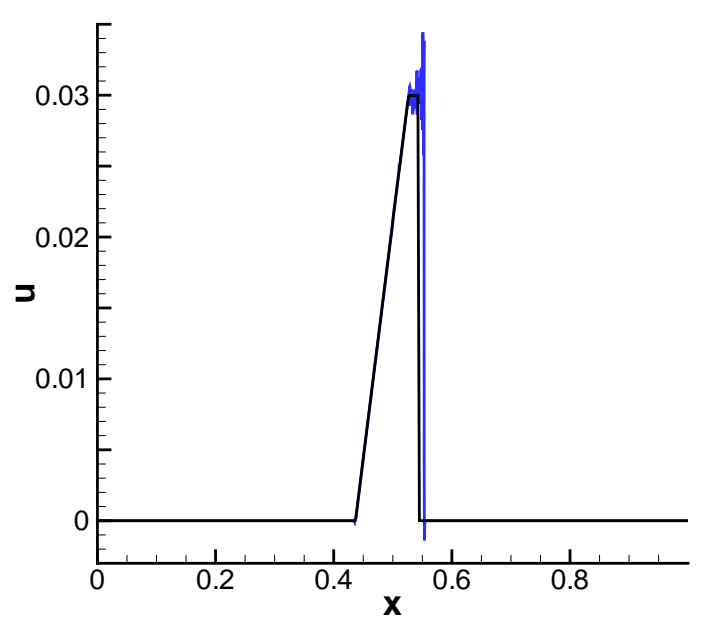

(c) velocity $u$

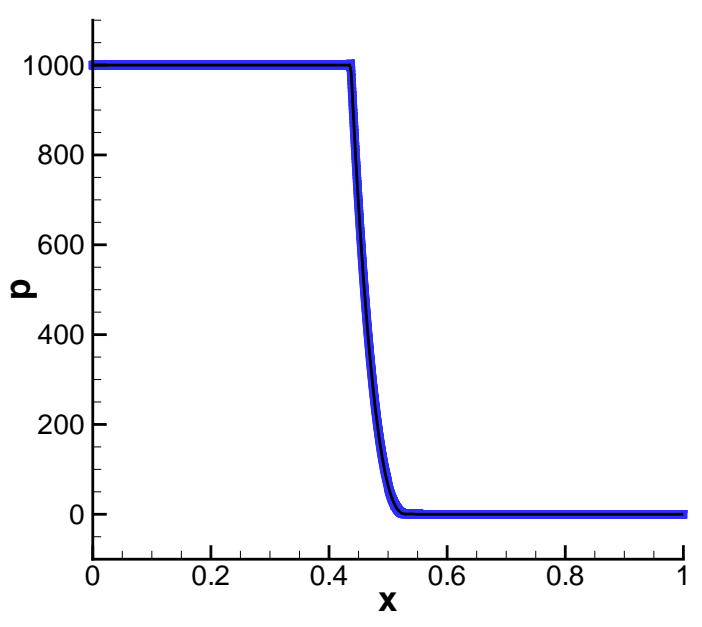

(b) pressure $p$

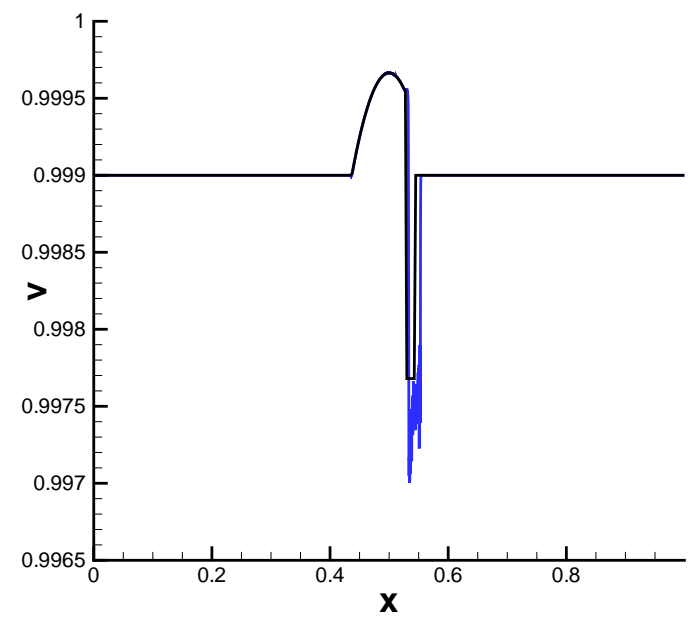

(d) velocity $v$

Figure 5.8: Example 5.1.5: One-dimensional Riemann problem with non-zero transverse velocity $v=0.999$ at $T=0.6$, approximated by the third-order RKDG method with the BP limiter. The approximations of the $u, v, p$ and $\rho$ on 3200 cells are presented. The blue solid lines are the numerical results and the black ones are the exact solutions.

present the contours as well as the cuts along the $x=y$ line of the numerical approximation for $\rho, p$ and $\sqrt{u^{2}+v^{2}}$. We see that as for the 1D counterpart, despite of small oscillations, all the structures: the shock, contact continuity and rarefaction, are well resolved and the physical bounds are preserved.

Example 5.2.3 (Two-dimensional Riemann problem). The two dimensional Riemann prob- 


\begin{tabular}{c|c|c|c|c|c}
\hline$k$ & $h$ & $L^{2}$ error & order & $L^{\infty}$ error & order \\
\hline \multirow{6}{*}{2} & $1 / 10$ & $4.53 \mathrm{e}-2$ & - & $1.98 \mathrm{e}-1$ & - \\
& $1 / 20$ & $2.61 \mathrm{e}-3$ & 4.11 & $1.64 \mathrm{e}-2$ & 3.60 \\
& $1 / 40$ & $2.97 \mathrm{e}-4$ & 3.14 & $1.65 \mathrm{e}-3$ & 3.31 \\
& $1 / 80$ & $3.84 \mathrm{e}-5$ & 2.95 & $2.16 \mathrm{e}-4$ & 2.93 \\
& $1 / 160$ & $5.13 \mathrm{e}-6$ & 2.91 & $2.75 \mathrm{e}-5$ & 2.97 \\
\hline
\end{tabular}

Table 5.2: Example 5.1.1: Two-dimensional accuracy test at $T=0.2$ for the third-order RKDG method with the BP limiter. $k$ is the degree of polynomial and $h$ is the mesh size.

lem involves the interactions of elementary waves, which initially separate four constant states. In [7], the authors extended the 2-D Riemann problem from the classical Newtonian hydrodynamics [35] to the relativistic flows. We take the same initial condition as in [7].

$$
\left(\rho_{0}, u_{0}, v_{0}, p_{0}\right)= \begin{cases}\left(0.1,0.0,0.0,10^{-2}\right), & x, y>0 \\ (0.1,0.99,0.0,1.0), & x<0<y \\ (0.5,0.0,0.0,1.0), & x, y<0 \\ (0.1,0.0,0.99,1.0), & y<0<x\end{cases}
$$

and outflow boundary condition is set everywhere.

Figure 5.10 shows the numerical approximation at $T=0.7$ on the $400 \times 400$ mesh. Since we only apply the BP limiter without any non-oscillatory technique, there are oscillations around the stationary contact discontinuity and the up-right moving shocks. Despite of this, the structure of the solution matches the results in $[54,7,24]$.

Example 5.2.4 (Axisymmetric relativistic jet). The last example is to apply our boundpreserving DG method to the simulation of the relativistic axisymmetric jet. We consider the equations in the cylindrical coordinates, (4.1) and (4.2). The numerical simulation, morphology and the dynamics of axisymmetric relativistic jets have been well studied in the literature $[10,23]$. We first consider the C2 model in [23]. In order to compare the results, we use the same parameters as those in [23]. The initial states are assigned to be

$$
\left(\rho, v_{z}, v_{r}, p\right)=\left(1.0,0.0,0.0,1.70305 \times 10^{-4}\right), \quad(r, z) \in[0,10] \times[0,20]
$$

Gas with density $\rho^{i n}=0.01$, pressure $p^{i n}=1.70305 \times 10^{-4}$ and velocity $v_{r}^{i n}=0.99$ is injected through the inlet part $r<1$. The classical Mach number of this model is $M_{b}=6$ and the 
corresponding relativistic Mach number is $\mathcal{M}_{b}=42$. In Figure 5.11, we show the numerical results at $T=21$ and $T=35$ respectively.

Next, we consider the C3 model in [23]. The initial states are

$$
\left(\rho, v_{z}, v_{r}, p\right)=\left(1.0,0.0,0.0,1.7355 \times 10^{-4}\right), \quad(r, z) \in[0,15] \times[0,25]
$$

The jet material has states $\left(\rho^{i n}, v_{z}^{i n}, v_{r}^{i n}, p^{i n}\right)=\left(0.01,0.999,0.0,1.7355 \times 10^{-4}\right)$. The relativistic Mach number of this model is around 132. The simulation result at $T=30$ is presented in Figure 5.12.

The proposed method behaves robustly for both of these highly relativistic jets, especially for the second one. The average speed of the jet head is 0.43 for the C2 model and 0.71 for the C3 model. These match the theoretical estimates in [23], which are 0.42 and 0.70 respectively. Very steady cocoon can be observed in the early stage of the evolution (Figure $5.11, T=20)$ and it eventually evolves into large vortices moving backwards. Due to the larger Lorentz factor, the cocoon of the C3 model is less prominent and thinner than that of the $\mathrm{C} 2$ one. We believe that, due to the high resolution property of the DG method, the Kelvin-Helmholtz instabilities in the cocoon, which is caused by the discontinuity between the shocked jet material and shocked medium material, are well resolved in both tests. Moreover, there is no "carbuncle" artifact generated. This pathological phenomena was first discussed in [30] for gas dynamics and was discussed and addressed for the RHD problem in $[9]$.

\section{Concluding remarks}

In this paper, we have addressed the robustness issues of the discontinuous Galerkin (DG) methods to solve relativistic hydrodynamics (RHD). A special bound-preserving limiter is constructed to obtain physically relevant numerical approximations without compromising

the high order accuracy. Moreover, we can prove the $L^{1}$-stability of the DG scheme with this limiter. Numerical experiments are given to demonstrate the good performance of the DG 
scheme. The bound-preserving limiter is very simple and inexpensive, and can be applied to any high order finite volume or DG base schemes. In the future, we plan to study other possible bound-preserving first order building blocks for RHD, and to generalize the boundpreserving technique to relativistic magnetohydrodynamics.

\section{A A proof for Lemma 2.1}

In this section, we will prove Lemma 2.1. For simplicity, we only study the proof for $H^{-}$, as the proof for $H^{+}$can be obtained along the same line. Recall that $\mathbf{w}=(D, m, E)^{T} \in G_{1}$ and $\mathbf{f}(\mathbf{w})=(D u, m u+p, m)^{T}$. Therefore,

$$
\alpha_{f} \mathbf{H}^{-}\left(\mathbf{w}, \alpha_{f}\right)=\alpha_{f} \mathbf{w}-\mathbf{f}(\mathbf{w})=\left(\left(\alpha_{f}-u\right) D,\left(\alpha_{f}-u\right) m-p, \alpha_{f} E-m\right)^{T} .
$$

It is easy to see that to obtain $\mathbf{H}^{-} \in G_{1}$, we need $\alpha_{f} \mathbf{H}^{-} \in G_{1}$, i.e.

$$
\begin{aligned}
\alpha_{f} & \geq \alpha_{1}=u, \\
\alpha_{f} & \geq \alpha_{2}=\frac{m}{E}, \\
\left(\alpha_{f} E-m\right)^{2} & \geq\left(\left(\alpha_{f}-u\right) m-p\right)^{2}+\left(\left(\alpha_{f}-u\right) D\right)^{2} .
\end{aligned}
$$

Define $q\left(\alpha_{f}\right)=\left(\alpha_{f} E-m\right)^{2}-\left(\left(\alpha_{f}-u\right) m-p\right)^{2}-\left(\left(\alpha_{f}-u\right) D\right)^{2}$. By using (1.3)-(1.6), and define $\beta_{f}=\alpha_{f}-u$, and $\tau=(\Gamma-1) / \Gamma$, we have

$$
\begin{aligned}
q\left(\alpha_{f}\right) & =\rho^{2}\left[\left(\beta_{f} h \gamma^{2}-\alpha_{f} \tau(h-1)\right)^{2}-\beta_{f}^{2} \gamma^{2}-\left(\beta_{f} h \gamma^{2} u-\tau(h-1)\right)^{2}\right] \\
& =\rho^{2}(h-1)\left[\beta_{f}^{2} \gamma^{2}(h+1)+\left(\alpha_{f}^{2}-1\right) \tau^{2}(h-1)-2 \alpha_{f} \beta_{f} h \tau \gamma^{2}+2 \beta_{f} h \tau \gamma^{2} u\right] \\
& =\rho^{2}(h-1)\left[\beta_{f}^{2} \gamma^{2}(h+1)+\left(\alpha_{f}^{2}-1\right) \tau^{2}(h-1)-2 \beta_{f}^{2} h \tau \gamma^{2}\right] \\
& =\rho^{2}(h-1)\left[A_{2} \alpha_{f}^{2}-2 A_{1} \alpha_{f}+A_{0}\right]
\end{aligned}
$$

where

$$
\begin{aligned}
& A_{2}=\gamma^{2}(h+1-2 h \tau)+\tau^{2}(h-1), \\
& A_{1}=u \gamma^{2}(h+1-2 h \tau),
\end{aligned}
$$




$$
A_{0}=u^{2} \gamma^{2}(h+1-2 h \tau)-\tau^{2}(h-1)
$$

Since $1 \leq \Gamma \leq 2$, then $0 \leq \tau \leq 1 / 2$. It is easy to check that

$$
A_{2} \geq 0, \quad q(1) \geq 0, \quad q(u) \leq 0, \quad q(-1) \geq 0
$$

To obtain (A.3), we can take

$$
\alpha_{f} \geq \alpha_{3}=\frac{A_{1}+\sqrt{\left(A_{1}\right)^{2}-A_{2} A_{0}}}{A_{2}} .
$$

If $u<0$, then $\alpha_{3} \geq 0 \geq \alpha_{1} \geq \alpha_{2}$. If $u \geq 0$, then $\alpha_{3} \geq \alpha_{2} \geq \alpha_{1} \geq 0$. Therefore, we have $H^{-} \in G_{1}$ under (A.7). Similarly, we can define

$$
\begin{aligned}
& A_{2}^{\prime}=\gamma^{2}(h+1-2 h \tau)+\tau^{2}(h-1)=A_{2}, \\
& A_{1}^{\prime}=-u \gamma^{2}(h+1-2 h \tau)=-A_{1}, \\
& A_{0}^{\prime}=u^{2} \gamma^{2}(h+1-2 h \tau)-\tau^{2}(h-1)=A_{0} .
\end{aligned}
$$

to obtain the lower bound of $\alpha_{f}$ :

$$
\alpha_{f} \geq \frac{A_{1}^{\prime}+\sqrt{\left(A_{1}^{\prime}\right)^{2}-A_{2}^{\prime} A_{0}^{\prime}}}{A_{2}^{\prime}} .
$$

(A.7) and (A.8) imply

$$
\alpha_{f} \geq \frac{\left|A_{1}\right|+\sqrt{\left(A_{1}\right)^{2}-A_{2} A_{0}}}{A_{2}},
$$

where $A_{0}, A_{1}$ and $A_{2}$ are given in (A.4)-(A.6).

\section{B A proof for Lemma 4.1}

In this section, we will prove Lemma 4.1. It is easy to see that

$$
\widetilde{\mathbf{H}}_{s}(\mathbf{w}, r, \Delta t)=(r D, r m+\Delta t p, r n, r E)^{T} .
$$

Define

$$
q(\mathbf{w}, r, \Delta t)=(r E)^{2}-(r D)^{2}-(r m+\Delta t p)^{2}-(r n)^{2}
$$


Since $r>0$, we only need to find $\Delta t$ such that $q(\mathbf{w}, r, \Delta t) \geq 0$. It is not difficult to check that

$$
\begin{aligned}
E & =\left(\frac{\Gamma}{\sigma}-1\right) p \\
m & =\frac{u \Gamma}{\sigma} p \\
n & =\frac{v \Gamma}{\sigma} p \\
D & =\frac{\gamma \Gamma}{(\Gamma-1)(h-1)} p,
\end{aligned}
$$

where $\sigma=\frac{(\Gamma-1)(h-1)}{h \gamma^{2}}$. Therefore, we have

$$
\begin{aligned}
q(\mathbf{w}, r, \Delta t) & =(r E)^{2}-(r D)^{2}-(r m+\Delta t p)^{2}-(r n)^{2} \\
& =p^{2}\left[\left(\frac{r \Gamma}{\sigma}-r\right)^{2}-\left(\frac{r \gamma \Gamma}{(\Gamma-1)(h-1)}\right)^{2}-\left(\frac{u \Gamma r}{\sigma}+\Delta t\right)^{2}-\left(\frac{r \Gamma v}{\sigma}\right)^{2}\right] \\
& =r^{2} p^{2}\left[\frac{\Gamma^{2}}{\sigma^{2} \gamma^{2}}+1-\frac{2 \Gamma}{\sigma}-\frac{\Delta t^{2}}{r^{2}}-\frac{2 u \Gamma}{\sigma} \frac{\Delta t}{r}-\left(\frac{\Gamma \gamma}{(\Gamma-1)(h-1)}\right)^{2}\right] \\
& =-r^{2} p^{2}\left(\frac{\Delta t^{2}}{r^{2}}+2 A \frac{\Delta t}{r}+B\right),
\end{aligned}
$$

where

$$
A=\frac{u \Gamma h \gamma^{2}}{(\Gamma-1)(h-1)}
$$

and

$$
B=\frac{\Gamma h \gamma^{2}(\Gamma-2)-\Gamma^{2} \gamma^{2}}{(\Gamma-1)^{2}(h-1)}-1<-1
$$

Let $q(\mathbf{w}, r, \Delta t) \geq 0$, we need

$$
\Delta t \leq\left(\sqrt{A^{2}-B}-A\right) r .
$$

\section{References}

[1] J. Centrella and J.R. Wilson, Planar numerical cosmology II: the difference equations and numerical tests, Astrophysics Journal Supplementary Series, 54 (1984), 229-249. 
[2] B. Cockburn, S. Hou and C.-W. Shu, The Runge-Kutta local projection discontinuous Galerkin finite element method for conservation laws IV: the multidimensional case, Mathematics of Computation, 54 (1990), 545-581.

[3] B. Cockburn, S.-Y. Lin and C.-W. Shu, TVB Runge-Kutta local projection discontinuous Galerkin finite element method for conservation laws III: one-dimensional systems, Journal of Computational Physics, 84 (1989), 90-113.

[4] B. Cockburn and C.-W. Shu, TVB Runge-Kutta local projection discontinuous Galerkin finite element method for conservation laws II: general framework, Mathematics of Computation, 52 (1989), 411-435.

[5] B. Cockburn and C.-W. Shu, The Runge-Kutta discontinuous Galerkin method for conservation laws V: multidimensional systems, Journal of Computational Physics, 141 (1998), 199-224.

[6] P. Colella and P. R. Woodward, The piecewise parabolic method (PPM) for gasdynamical simulations, Journal of Computational Physics, 54 (1984), 174-201.

[7] L. Del Zanna and N. Bucciantini, An efficient shock-capturing central-type scheme or multidimensional relativistic flows I. Hydrodynamics, Astronomy and Astrophysics, 390 (2002), 1177-1186.

[8] A. Dolezal and S. Wong, Relativistic hydrodynamics and essentially non-oscillatory shock capturing scheme, Journal of Computational Physics, 120 (1995), 266-277.

[9] R. Donat, J.A. Font, J.M. Ibáñez and A. Marquina, A flux-split algorithm applied to relativistic flows, Journal of Computational Physics, 146 (1998), 58-81.

[10] G.C. Duncan and P.A. Hughes, Simulations of relativistic extragalactic jets, The Astrophysical Journal, 436 (1994), L119-L122 
[11] F. Eulderink, Numerical Relativistic Hydrodynamics, PhD Thesis, Rijksuniverteit te Leiden, Leiden, Netherlands, 1993.

[12] F. Eulderink and G. Mellema, Special relativistic jet collimation by inertial confinement, Astronomy and Astrophysics, 284 (1994), 654-662.

[13] S. Gottlieb, C.-W. Shu and E. Tadmor, Strong stability-preserving high-order time discretization methods, SIAM Review, 43 (2001), 89-112.

[14] P. He and H. Tang, An adaptive moving mesh method for two-dimensional relativistic hydrodynamics, Communications in Computational Physics, 11 (2012), 114-146.

[15] X. Hu, N. Adams and C.-W. Shu, Positivity preserving method for high-order conservative schemes solving compressible Euler equations, Journal of Computational Physics, 242 (2013), 169-180.

[16] M. Kunik, S. Qamar and G. Warnecke, Kinetic schemes for the relativistic gas dynamics, Numerische Mathematik, 97 (2004), 159-191.

[17] A. Lucas-Serrano, J. Font, J.M. Ibáñez and J. Martí, Assessment of a high-resolution central scheme for the solution of the relativistic hydrodynamics equations, Astronomy and Astrophysics, 428 (2004), 703-715.

[18] A. Marquina, J. Martí, J. Ibáñez, J. Miralles and R. Donat, Ultrarelativistic hydrodynamics - High-resolution shock-capturing methods, Astronomy and Astrophysics, 258 (1992), 566-571.

[19] J. Martí, J. Ibáñez and J.A. Miralles, Numerical relativistic hydrodynamics: Local characteristic approach, Physics Review D, 43 (1991), 3794.

[20] J. Martí and E. Müller, The analytical solution of the Riemann problem in relativistic hydrodynamics, Journal of Fluid Mechanics, 258 (1994), 317-333. 
[21] J. Martí and E. Müller, Extension of the piecewise parabolic method to one-dimensional relativistic hydrodynamics, Journal of Computational Physics, 123 (1996), 1-14.

[22] J. Martí and E. Müller, Numerical hydrodynamics in special relativity, Living Review in Relativity, 6 (2003), 7.

[23] J. Martí, E. Müller, J. Font, J. Ibáñez and A. Marquina, Morphology and dynamics of relativistic jets, The Astrophysical Journal, 479 (1997), 151-163.

[24] A. Mignone and G. Bodo, An HLLC Riemann solver for relativistic flows - I. Hydrodynamics, Monthly Notices of the Royal Astronomical Society, 364 (2005), 126-136.

[25] A. Mignone, T. Plewa and G. Bodo, The piecewise parabolic method for multidimensional relativistic fluid dynamics, The Astrophysical Journal Supplement Series, 160 (2005), 199-220.

[26] J. von Neumann and R.D. Richtmyer, A method for the numerical calculation of hydrodynamical shocks, Journal of Applied Physics, 21 (1950), 232-247.

[27] B. O’Shea, G. Bryan, J. Bordner, M. Norman, T. Abel, R. Harkness and A. Kritsuk, Introducing Enzo, an AMR cosmology application, T. Plewa, T. Linde and V. G. Weirs (Eds.), Springer Lecture Notes in Computational Science and Engineering, 2004.

[28] S. Qamar and G. Warnecke, A high-order kinetic flux-splitting method for the relativistic magnetohydrodynamics, Journal of Computational Physics, 205 (2005), 182-204.

[29] J.X. Qiu and C.-W. Shu, RungeKutta discontinuous Galerkin method using WENO limiters, SIAM Journal on Scientific Computing, 26 (2005), 907-929.

[30] J. Quirk, A contribution to the great Riemann solver debate, International Journal for Numerical Methods in Fluids, 18 (1994), 555-574. 
[31] D. Radice and L. Rezzolla, Discontinuous Galerkin methods for general-relativistic hydrodynamics: formulation and application to spherical symmetric spacetimes, Physical Review D, 84 (2011), 024010.

[32] W.H. Reed and T.R. Hill, Triangular mesh methods for the Neutron transport equation, Los Alamos Scientific Laboratory Report LA-UR-73-479, Los Alamos, NM, 1973.

[33] R.D. Richtmyer and K.W. Morton Difference Methods for Initial-value Problems, WileyInterscience, New York, 1967.

[34] V. Schneider, U. Katscher, D. Rischke, B. Waldhauser, J. Maruhn and C. Munz, New algorithm for ultra-relativistic numerical hydrodynamics, Journal of Computational Physics, 105 (1993), 92-107.

[35] C.W. Schulz-Rinne, J.P. Collins and H.M. Glaz, Numerical solution of the Riemann problem for two dimensional gas dynamics, SIAM Journal on Scientific Computing, 14 (1993), 1394-1414.

[36] C.-W. Shu, TVB uniformly high-order schemes for conservation laws, Mathematics of Computation, 49 (1987), 105-121.

[37] C.-W. Shu, Total-variation-diminishing time discretizations, SIAM Journal on Scientific and Statistical Computing, 9 (1988), 1073-1084.

[38] C.-W. Shu and S. Osher, Efficient implementation of essentially non-oscillatory shockcapturing schemes, Journal of Computational Physics, 77 (1988), 439-471.

[39] A. Taub, Relativistic Rankine-Hugoniot equations, Physical Review, 74 (1948), 328.

[40] A. Tchekhovskoy, J. McKinney and R. Narayan, A WENO-based general relativistic numerical scheme - I. Hydrodynamics, Monthly Notices of the Royal Astronomical Society, 379 (2007), 469-497. 
[41] R. Teyssier, Cosmology hydrodynamics with adaptive mesh refinement. A new high resolution code called RAMSES, Astronomy and Astrophysics, 385 (2002), 337-364.

[42] C. Wang, X. Zhang, C.-W. Shu and J. Ning, Robust high order discontinuous Galerkin schemes for two-dimensional gaseous detonations, Journal of Computational Physics, 231 (2012), 653-665.

[43] P. Wang, T. Abel and W. Zhang, Relativistic hydrodynamics flows using spatial and temporal adaptive structured mesh refinement, The Astrophysical Journal Supplement Series, 176 (2008), 467-483.

[44] J.R. Wilson, Numerical study of fluid flow in a Kerr space, Astrophysics Journal, 173 (1972), 431-438.

[45] J.R. Wilson, A numerical method for relativistic hydrodynamics, in L.L. Smarr (ed.), Sources of Gravitational Radiation, Cambridge University Press, Cambridge, U.K., 1979, 423-446.

[46] K. Wu and H. Tang, Finite volume local evolution Galerkin method for two-dimensional relativistic hydrodynamics, Journal of Computational Physics, 256 (2014), 277-307.

[47] K. Wu and H. Tang, High-order accurate physical-constraints-preserving finite difference WENO schemes for special relativistic hydrodynamics, Journal of Computational Physics, 298 (2015), 539-564.

[48] Y. Xing, X. Zhang and C.-W. Shu, Positivity preserving high order well balanced discontinuous Galerkin methods for the shallow water equations, Advances in Water Resources, v33 (2010), 1476-1493.

[49] Z.F. Xu, Parametrized maximum principle preserving flux limiters for high order schemes solving hyperbolic conservation laws: one-dimensional scalar problem, Mathematics of Computation, 83 (2014), 310-331. 
[50] Y. Yang and C.-W. Shu, Discontinuous Galerkin method for hyperbolic equations involving $\delta$-singularities: negative-order norm error estimates and applications, Numerische Mathematik, 124, (2013), 753-781.

[51] Y. Yang, D. Wei and C.-W. Shu, Discontinuous Galerkin method for Krause's consensus models and pressureless Euler equations, Journal of Computational Physics, 252 (2013), 109-127.

[52] Z. Yang, P. He and H. Tang, A direct Eulerian GRP scheme for relativistic hydrodynamics: One-dimensional case, Journal of Computational Physics, 230 (2011), 7964-7987.

[53] Z. Yang and H. Tang, A direct Eulerian GRP scheme for relativistic hydrodynamics: Two-dimensional case, Journal of Computational Physics, 231 (2012), 2116-2139.

[54] W. Zhang and A. MacFadyen, RAM: A relativistic adaptive mesh refinement hydrodynamics code, The Astrophysical Journal Supplement Series, 164 (2006), 255-279.

[55] X. Zhang and C.-W. Shu, On maximum-principle-satisfying high order schemes for scalar conservation laws, Journal of Computational Physics, 229 (2010), 3091-3120.

[56] X. Zhang and C.-W. Shu, On positivity preserving high order discontinuous Galerkin schemes for compressible Euler equations on rectangular meshes, Journal of Computational Physics, 229 (2010), 8918-8934.

[57] X. Zhang and C.-W. Shu, Positivity-preserving high order discontinuous Galerkin schemes for compressible Euler equations with source terms, Journal of Computational Physics, 230 (2011), 1238-1248.

[58] X. Zhang and C.-W. Shu, Maximum-principle-satisfying and positivity-preserving high order schemes for conservation laws: Survey and new developments, Proceedings of the Royal Society A, 467 (2011), 2752-2776. 
[59] X. Zhang and C.-W. Shu, A minimum entropy principle of high order schemes for gas dynamics equations, Numerische Mathematik, 121 (2012), 545-563.

[60] X. Zhang, Y. Xia and C.-W. Shu, Maximum-principle-satisfying and positivitypreserving high order discontinuous Galerkin schemes for conservation laws on triangular meshes, Journal of Scientific Computing, 50 (2012), 29-62.

[61] J. Zhao, P. He and H. Tang, Steger-Warming flux vector splitting method for special relativistic hydrodynamics, Mathematical Methods in the Applied Sciences, 37 (2014), 1003-1018.

[62] J. Zhao and H. Tang, RungeKutta discontinuous Galerkin methods with WENO limiter for the special relativistic hydrodynamics, Journal of Computational Physics, 242 (2013), $138-168$. 


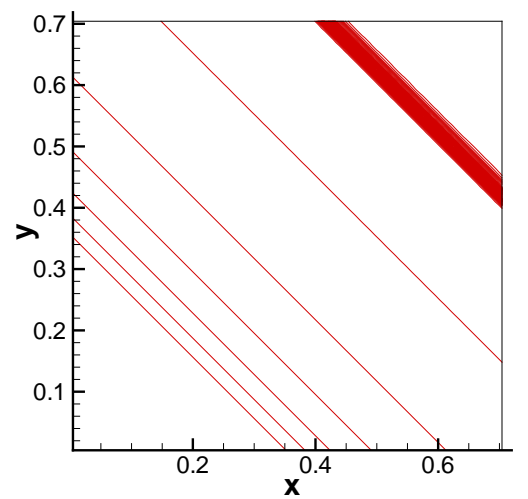

(a) 50 equally spaced contours of $\rho$ from 0 to 8

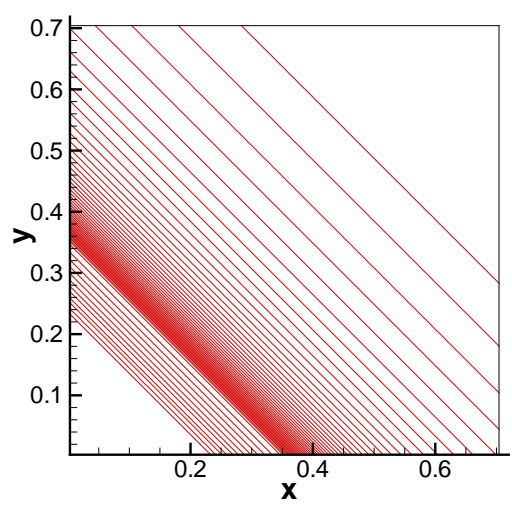

(c) 50 equally spaced contours of $p$ from 0 to 1000

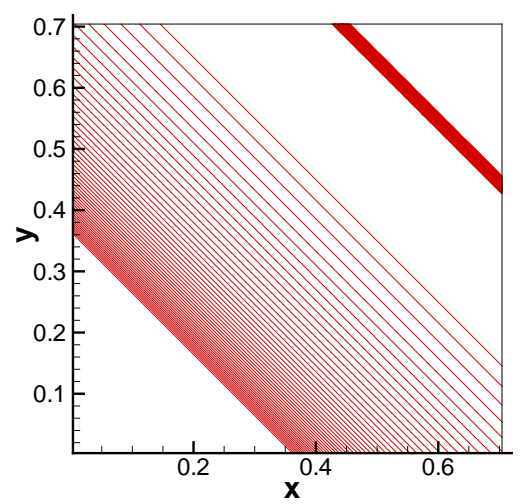

(e) 50 equally spaced contours of $\sqrt{u^{2}+v^{2}}$ from 0 to 1

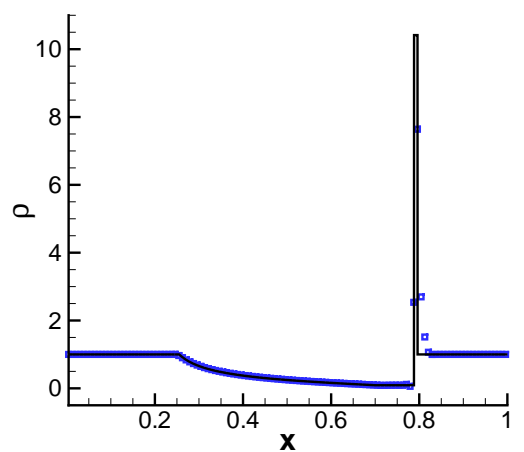

(b) cut along $x=y$

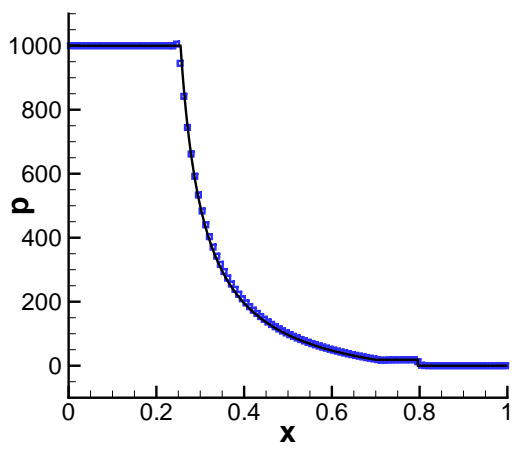

(d) cut along $x=y$

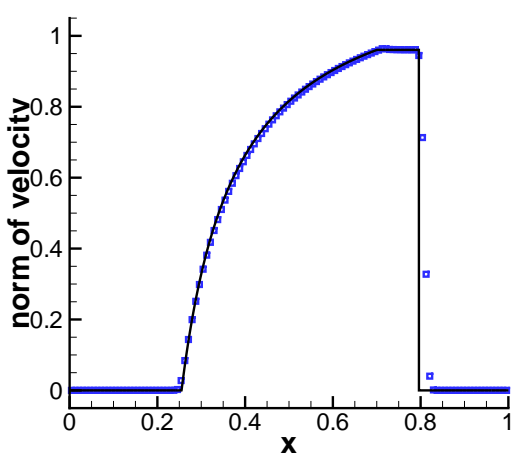

(f) cut along $x=y$

Figure 5.9: Example 5.2.2: Blast wave with initial condition (5.6) propagating in $\left[0, \frac{\sqrt{2}}{2}\right]^{2}$. At time $T=0.3$ on a $120 \times 120$ mesh. In the right column, solid lines are exact solution and the blue squares are the approximate ones. 


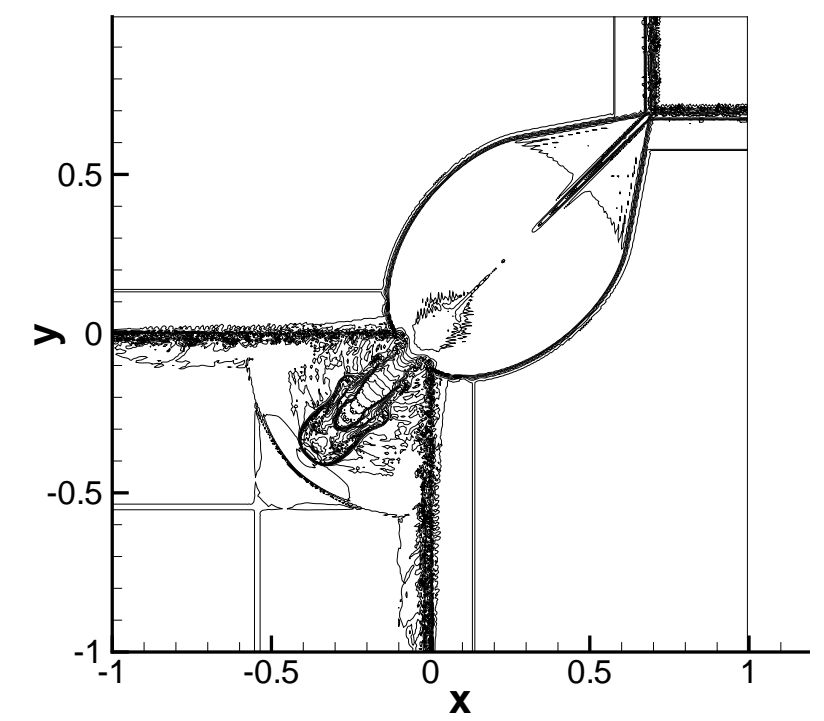

Figure 5.10: Example 5.2.3: $400 \times 400$ cells at $T=0.7$ approximated by the third-order RKDG method with the BP limiter. Thirty equally spaced contours of the logarithm of the proper density are plotted.

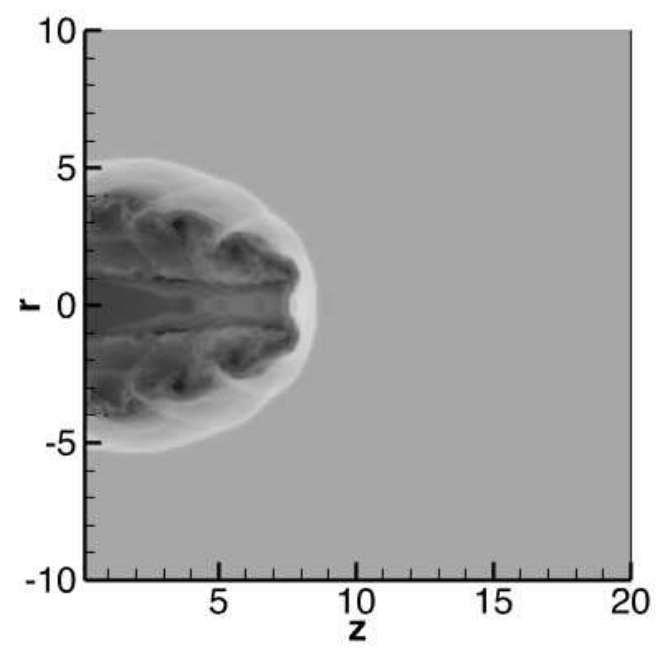

(a) $\mathrm{T}=20$

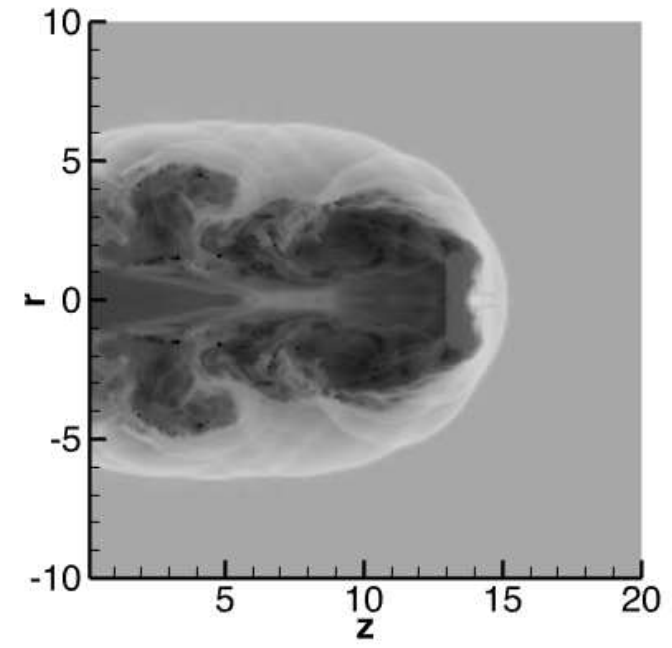

(b) $\mathrm{T}=35$

Figure 5.11: Example 5.7: Relativistic jets of model C2 with initial condition (5.7). The left panel is at $T=20$ and the right is at $T=35$. The resolution is 10 points per jet radius. 


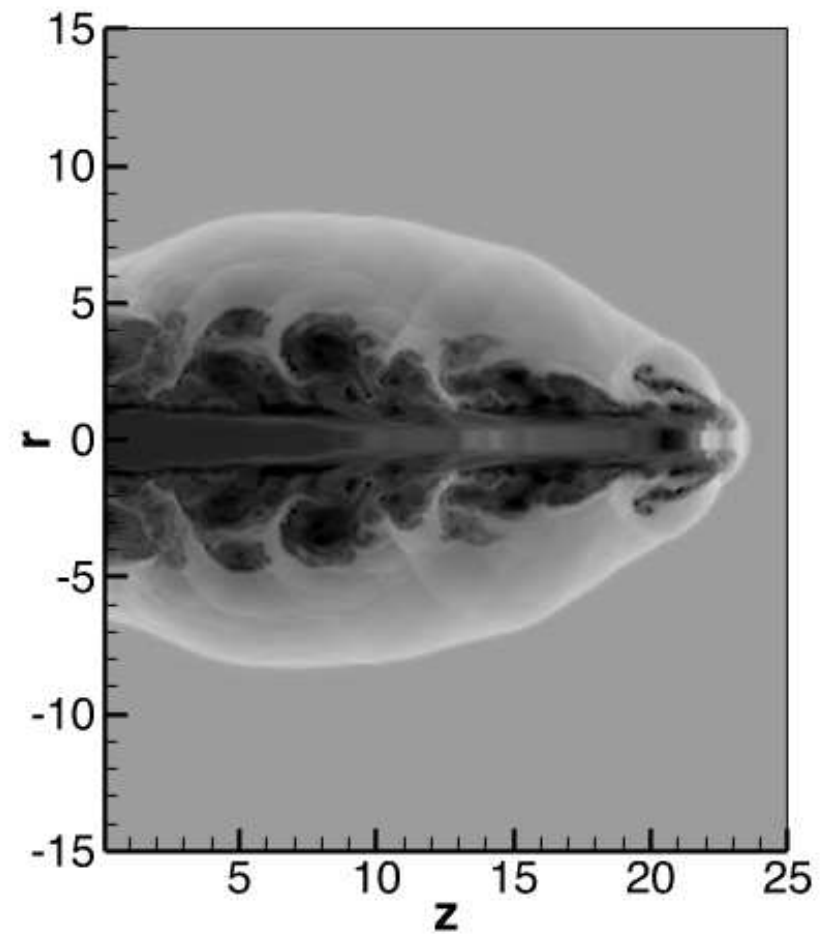

Figure 5.12: Example 5.2.4: Relativistic jet of the model C3 with initial condition (5.8) at $T=30$, approximated by RKDG with the BP limiter. The resolution is 10 points per jet radius. 